\title{
Soft thermal contributions to 3-loop gauge coupling
}

\section{Laine, ${ }^{a}$ P. Schicho ${ }^{a}$ and Y. Schröder ${ }^{b}$}

${ }^{a} A E C$, Institute for Theoretical Physics, University of Bern,

Sidlerstrasse 5, 3012 Bern, Switzerland

${ }^{b}$ Grupo de Cosmología y Partículas Elementales, Universidad del Bío-Bío, Casilla 447, Chillán, Chile

E-mail: laine@itp.unibe.ch, schicho@itp.unibe.ch, yschroder@ubiobio.cl

ABSTRACT: We analyze 3-loop contributions to the gauge coupling felt by ultrasoft ("magnetostatic") modes in hot Yang-Mills theory. So-called soft/hard terms, originating from dimension-six operators within the soft effective theory, are shown to cancel 1097/1098 of the IR divergence found in a recent determination of the hard 3-loop contribution to the soft gauge coupling. The remaining 1/1098 originates from ultrasoft/hard contributions, induced by dimension-six operators in the ultrasoft effective theory. Soft 3-loop contributions are likewise computed, and are found to be IR divergent, rendering the ultrasoft gauge coupling non-perturbative at relative order $\mathcal{O}\left(\alpha_{\mathrm{s}}^{3 / 2}\right)$. We elaborate on the implications of these findings for effective theory studies of physical observables in thermal QCD.

Keywords: Thermal Field Theory, Quark-Gluon Plasma, Resummation

ARXiv EPrint: 1803.08689 


\section{Contents}

1 Introduction 1

2 Form of EQCD 2

2.1 Super-renormalizable part 2

2.2 Dimension-six operators 4

2.3 Details on the determination of dimension-six coefficients 6

3 Overlapping soft/hard and ultrasoft/hard contributions $\quad 8$

3.1 1-loop results with dimension-six operators 9

$\begin{array}{ll}3.2 & \text { 2-loop results with dimension-six operators } \\ 3.3\end{array}$

$\begin{array}{ll}3.3 & \text { Contribution from dimension-six operators in MQCD } \\ \end{array}$

4 Soft and overlapping ultrasoft/soft contributions $\quad 14$

$\begin{array}{lll}4.1 & \text { Direct soft terms up to 3-loop level } & 14\end{array}$

$\begin{array}{lll}4.2 & \text { Contribution from dimension-six operators in MQCD } & 16\end{array}$

$\begin{array}{llr}5 & \text { Conclusions } & 17\end{array}$

$\begin{array}{lr}\text { A Spacetime and colour tensors } & 18\end{array}$

$\begin{array}{lr}\text { B Basic sum-integrals } & 19\end{array}$

$\begin{array}{ll}\text { C Dimension-six vertices in the } S / T \text { basis } & 20\end{array}$

$\begin{array}{ll}\text { D Basic vacuum integrals } & 23\end{array}$

E Details concerning 2-loop and 3-loop results 24

\section{Introduction}

Dimensionally reduced ("3d") thermal effective theories, originally conceived for studying thermodynamics and phase transitions in non-Abelian gauge theories [1-3], and still used for that purpose in the context of weak interactions (cf. e.g. refs. [4, 5] for recent work and references), have been reinvigorated in another context some time ago. Indeed, quite remarkably, they also turn out to determine soft contributions to real-time lightcone observables [6]. As examples, they can be used for estimating the so-called transverse collision kernel related to jet quenching in a hot QCD plasma [7, 8]; soft parts of the photon and dilepton production rates from a QCD plasma [9, 10]; and the interaction rate experienced by neutrinos in an electroweak plasma [11]. Following standard terminology, we refer to 
the "soft" effective theory as EQCD, whereas the "ultrasoft" theory containing only the magnetostatic modes is called MQCD (cf. e.g. refs. [12-15]). The latter has been argued to give e.g. the leading non-perturbative contribution to jet quenching [16].

In the QCD context it is known, however, that EQCD fails to describe the full theory close to the phase transition or crossover temperature $\left(T_{\mathrm{c}}\right)$. This is obvious when light quarks are present: EQCD contains only gluonic degrees of freedom, and displays no remnant of the flavour symmetries that underlie the chiral transition. For pure-glue theory, the reason for the breakdown is more subtle. Even though the center symmetry that drives the transition in the imaginary-time formulation [17] is not explicit in EQCD, remnants of it are generated dynamically [18]. However the dynamical re-generation is incomplete, and a $3 d$ lattice study in which soft EQCD dynamics was treated non-perturbatively did not achieve satisfactory agreement with thermodynamic functions obtained from full $4 \mathrm{~d}$ lattice simulations [19].

One purpose of this paper is to demonstrate analytically that power-suppressed dimension-six operators, truncated from the super-renormalizable EQCD description, play an essential role in soft and ultrasoft observables, and are therefore a likely culprit for EQCD's failure close to $T_{\mathrm{c}}$. More concretely, we determine the MQCD gauge coupling in terms of the EQCD gauge coupling and mass parameter up to 3-loop level, including the 1- and 2-loop contributions of all dimension-six operators; the result is contained in eqs. (3.13), (3.14) and (4.4).

Our presentation is organized as follows. After reviewing the form of EQCD and rederiving the coefficients of its dimension-six operators in section 2, we determine overlapping soft/hard and ultrasoft/hard contributions to the ultrasoft gauge coupling in section 3 . In terms of four-dimensional Yang-Mills we go up to 3-loop level; this implies 2-loop level in effects originating from dimension-six operators, which are themselves generated by 1-loop diagrams. A 3-loop computation of soft effects, as well as of overlapping ultrasoft/soft contributions, is presented in section 4, whereas conclusions are collected in section 5 . Spacetime and colour tensors, tensor-like 1-loop sum-integrals, Feynman rules related to dimension-six operators, $d$-dimensional vacuum integrals, and some lengthier results, are collected in five appendices, respectively.

\section{Form of EQCD}

\section{$2.1 \quad$ Super-renormalizable part}

The super-renormalizable truncation of the dimensionally reduced "electrostatic" QCD, called EQCD, is defined by the action

$$
\begin{aligned}
S_{\mathrm{EQCD}}[A] \equiv \int_{X}\{ & \frac{1}{4} F_{i j}^{a} F_{i j}^{a}+\frac{1}{2} \mathcal{D}_{i}^{a b} A_{0}^{b} \mathcal{D}_{i}^{a c} A_{0}^{c}+\frac{m_{\mathrm{E}}^{2}}{2} A_{0}^{a} A_{0}^{a} \\
& \left.+\frac{\lambda_{\mathrm{E}}}{4} X^{a b c d} A_{0}^{a} A_{0}^{b} A_{0}^{c} A_{0}^{d}+\frac{\kappa_{\mathrm{E}}}{4} A_{0}^{a} A_{0}^{a} A_{0}^{b} A_{0}^{b}\right\} .
\end{aligned}
$$

Here $\int_{X} \equiv \frac{1}{T} \int_{\mathbf{x}}, F_{i j}^{a} \equiv \partial_{i} A_{j}^{a}-\partial_{j} A_{i}^{a}+g_{\mathrm{E}} f^{a b c} A_{i}^{b} A_{j}^{c}, \mathcal{D}_{i}^{a b} \equiv \delta^{a b} \partial_{i}-g_{\mathrm{E}} f^{a b c} A_{i}^{c}, A_{0}^{a}$ is an adjoint scalar, $X^{a b c d}$ is defined in eq. (A.6), Latin indices take values $i, j \in\{1, \ldots, d\}$, we have 
in mind $d \equiv 3-2 \epsilon$, and repeated indices are summed over. We employ a convention in which the fields $A_{i}^{a}$ and $A_{0}^{a}$ have the same dimensionality as in four-dimensional Yang-Mills theory. Then explicit factors of $1 / T$ and $T$ appear in configuration and momentum space integration measures, respectively, where $T$ is the temperature.

Focussing on pure $\mathrm{SU}\left(N_{\mathrm{c}}\right)$ gauge theory, ${ }^{1}$ i.e. suppressing contributions proportional to the number of fermion flavours $\left(N_{\mathrm{f}}\right)$, the parameters appearing in eq. (2.1) have the expressions

$$
\begin{aligned}
& m_{\mathrm{E}}^{2}=g_{\mathrm{B}}^{2} N_{\mathrm{c}} \mathcal{F}_{P}^{\prime} \frac{(d-1)^{2}}{P^{2}}+\mathcal{O}\left(g_{\mathrm{B}}^{4}\right), \\
& g_{\mathrm{E}}^{2}=g_{\mathrm{B}}^{2}\left[1+g_{\mathrm{B}}^{2} N_{\mathrm{c}} \mathcal{F}_{P}^{\prime} \frac{25-d}{6 P^{4}}+\mathcal{O}\left(g_{\mathrm{B}}^{4}\right)\right], \\
& \lambda_{\mathrm{E}}=g_{\mathrm{B}}^{4}(d-1)^{2}(3-d) \sum_{P}^{\prime} \frac{1}{3 P^{4}}+\mathcal{O}\left(g_{\mathrm{B}}^{6}\right), \quad \kappa_{\mathrm{E}}=\mathcal{O}\left(g_{\mathrm{B}}^{4} N_{\mathrm{f}}\right),
\end{aligned}
$$

where $g_{\mathrm{B}}^{2}=g^{2} \mu^{2 \epsilon}\left(1+\mathcal{O}\left(g^{2}\right)\right)$ is the bare coupling of the original four-dimensional theory, $\mu$ is the scale parameter introduced in the context of dimensional regularization, and $g^{2} \equiv 4 \pi \alpha_{\mathrm{s}}$ is the renormalized coupling. By $\mathcal{K}_{P}^{\prime}$ we denote a sum-integral over $P$, with the prime indicating that the Matsubara zero mode is omitted. A 1-loop re-derivation of eqs. (2.2)(2.4) can be found as a side product of section 2.3; 2-loop expressions were obtained in ref. [20]; the 3-loop level has been reached for $m_{\mathrm{E}}^{2}[21]$ and $g_{\mathrm{E}}^{2}[22,23]$.

For our higher-loop computations in section 3 , it is helpful to express the dependence on $\lambda_{\mathrm{E}}$ and $\kappa_{\mathrm{E}}$ through the dimensionless combinations

$$
\begin{aligned}
\lambda & \equiv \frac{5 \lambda_{\mathrm{E}} N_{\mathrm{c}}}{4 g_{\mathrm{E}}^{2}}+\frac{\kappa_{\mathrm{E}}\left(N_{\mathrm{c}}^{2}+1\right)}{2 g_{\mathrm{E}}^{2} N_{\mathrm{c}}}, \\
\kappa_{1} & \equiv \frac{\lambda_{\mathrm{E}}\left(N_{\mathrm{c}}^{2}+36\right)}{2 g_{\mathrm{E}}^{2} N_{\mathrm{c}}}+\frac{10 \kappa_{\mathrm{E}}}{g_{\mathrm{E}}^{2} N_{\mathrm{c}}}, \\
\kappa_{2} & \equiv \frac{\lambda_{\mathrm{E}}^{2}\left(N_{\mathrm{c}}^{2}+36\right)}{4 g_{\mathrm{E}}^{4}}+\frac{10 \lambda_{\mathrm{E}} \kappa_{\mathrm{E}}}{g_{\mathrm{E}}^{4}}+\frac{2 \kappa_{\mathrm{E}}^{2}\left(N_{\mathrm{c}}^{2}+1\right)}{g_{\mathrm{E}}^{4} N_{\mathrm{c}}^{2}} .
\end{aligned}
$$

We note in passing that fundamental representation couplings often used in the literature, viz. $\lambda_{\mathrm{E}}^{(1)}\left(\operatorname{Tr}\left[A_{0}^{2}\right]\right)^{2}+\lambda_{\mathrm{E}}^{(2)} \operatorname{Tr}\left[A_{0}^{4}\right]$, are given by $\lambda_{\mathrm{E}}^{(1)}=3 \lambda_{\mathrm{E}} / 2+\kappa_{\mathrm{E}}$ and $\lambda_{\mathrm{E}}^{(2)}=\lambda_{\mathrm{E}} N_{\mathrm{c}} / 2$.

The theory can be renormalized through

$$
g_{\mathrm{E}}^{2}=g_{\mathrm{ER}}^{2} \mu^{2 \epsilon}+\delta g_{\mathrm{E}}^{2}, \quad m_{\mathrm{E}}^{2}=m_{\mathrm{ER}}^{2}+\delta m_{\mathrm{E}}^{2},
$$

and similarly for the scalar couplings. Within the super-renormalizable truncation, the counterterms take the forms $[24,25]$

$$
\delta g_{\mathrm{E}}^{2}=0, \quad \delta m_{\mathrm{E}}^{2}=\left(\frac{g_{\mathrm{ER}}^{2} N_{\mathrm{c}} T}{4 \pi}\right)^{2} \frac{\kappa_{2}-4 \lambda}{4 \epsilon} .
$$

\footnotetext{
${ }^{1}$ We omit fermions for simplicity because they carry non-zero Matsubara freqencies and thus generate no direct IR divergences. In other words they have no bearing on our conceptual discussion. If they were to be included, the expressions in eqs. (2.2)-(2.4), (2.18)-(2.20) and, most importantly, (2.11)-(2.12), would contain additional terms involving $N_{\mathrm{f}}$. Unfortunately the determination of the last of these effects entails an enormous practical effort, which we defer to future work.
} 
The starting point for our analysis is the 3-loop determination of $g_{\mathrm{E}}^{2}$ from fourdimensional Yang-Mills theory [22, 23]. It is helpful to display the result in the form of a background field effective action [26]. After gauge coupling and wave function renormalization through vacuum counterterms, refs. $[22,23]$ found an expression containing a logarithmic $(1 / \epsilon)$ divergence,

$$
\begin{aligned}
\Gamma_{\mathrm{EQCD}}^{(2)}[B]= & \frac{1}{2} B_{i}^{a}(q) B_{j}^{b}(r) \delta^{a b} \delta(q+r)\left(q^{2} \delta_{i j}-q_{i} q_{j}\right)\left(\mathcal{Z}_{B}+\delta \mathcal{Z}_{B}\right), \\
\mathcal{Z}_{B}= & 1-\frac{g^{2} N_{\mathrm{c}}}{(4 \pi)^{2}}\left[\frac{22}{3} \ln \left(\frac{\bar{\mu} e^{\gamma_{\mathrm{E}}}}{4 \pi T}\right)+\frac{1}{3}\right]-\frac{g^{4} N_{\mathrm{c}}^{2}}{(4 \pi)^{4}}\left[\frac{68}{3} \ln \left(\frac{\bar{\mu} e^{\gamma_{\mathrm{E}}}}{4 \pi T}\right)+\frac{341}{18}-\frac{10 \zeta_{3}}{9}\right] \\
& -\frac{g^{6} N_{\mathrm{c}}^{3}}{(4 \pi)^{6}}\left[\frac{748}{9} \ln ^{2}\left(\frac{\bar{\mu} e^{\gamma_{\mathrm{E}}}}{4 \pi T}\right)+\left(\frac{6608}{27}-\frac{10982 \zeta_{3}}{135}\right) \ln \left(\frac{\bar{\mu} e^{\gamma_{\mathrm{E}}}}{4 \pi T}\right)+(\text { finite })\right]+\mathcal{O}\left(g^{8}\right), \\
\delta \mathcal{Z}_{B}= & \frac{g^{6} N_{\mathrm{c}}^{3}}{(4 \pi)^{6}} \frac{61 \zeta_{3}}{5 \epsilon}+\mathcal{O}\left(g^{8}\right) .
\end{aligned}
$$

Here $\zeta_{n} \equiv \zeta(n)$ and $\bar{\mu}^{2} \equiv 4 \pi \mu^{2} e^{-\gamma_{\mathrm{E}}}$. The renormalized gauge coupling is given by $g_{\mathrm{ER}}^{2}=$ $g^{2} / \mathcal{Z}_{B}$, and the corresponding counterterm by $\delta g_{\mathrm{E}}^{2}=-g^{2} \mu^{2 \epsilon} \delta \mathcal{Z}_{B}+\mathcal{O}\left(g^{10}\right)$. We stress that eqs. (2.11) and (2.12) are gauge independent [27].

An essential technical goal of our investigation is to demonstrate how the divergence in eq. (2.12) is cancelled by overlapping soft/hard and ultrasoft/hard contributions, originating from dimension-six operators within EQCD and MQCD, respectively.

At this point we would like to clarify why such logarithmic divergences (which are "universal", i.e. present in any regularization scheme) originate first at 3-loop level. In three dimensions, 1-loop graphs may contain power divergences but no logarithmic divergences. Logarithmic divergences first originate at 2-loop level. However, within the super-renormalizable truncation of EQCD, they lead to the counterterms in eq. (2.9), i.e. the gauge coupling is finite. Divergences affecting the gauge coupling can only emerge when dimension-six operators are added to EQCD. Given that dimension-six operators are themselves generated by 1-loop diagrams, the divergences correspond to the 3-loop level in terms of the fundamental theory. In section 3 , where effects originating from integrating out the hard scale are considered, 3-loop level corresponds to the relative accuracy $O\left(g^{6}\right)$, whereas in section 4 , where effects originating from integrating out the soft scale are at focus, the expansion parameter is $\sim g$, and the 3-loop effects are of relative magnitude $O\left(g^{3}\right)$.

\subsection{Dimension-six operators}

The dimension-six operators that can be added to eq. (2.1) were determined in ref. [28]. We represent the operators as matrices in the adjoint representation. Letting Greek indices take values $\mu \in\{0, \ldots, d\}$, computing the coefficients at 1-loop level, and choosing to rephrase the gauge coupling as the same $g_{\mathrm{E}}$ as appears inside $F_{i j}^{a}$ and $\mathcal{D}_{i}^{a b}$, the dimension-six action 
can be written as

$$
\begin{aligned}
\delta S_{\mathrm{EQCD}}[A]=\sum_{P}^{\prime} \frac{2 g_{\mathrm{E}}^{2}}{P^{6}} \int_{X} \operatorname{tr} & \left\{c_{1}\left(D_{\mu} F_{\mu \nu}\right)^{2}+c_{2}\left(D_{\mu} F_{\mu 0}\right)^{2}\right. \\
& +i g_{\mathrm{E}}\left[c_{3} F_{\mu \nu} F_{\nu \rho} F_{\rho \mu}+c_{4} F_{0 \mu} F_{\mu \nu} F_{\nu 0}+c_{5} A_{0}\left(D_{\mu} F_{\mu \nu}\right) F_{0 \nu}\right] \\
& +g_{\mathrm{E}}^{2}\left[c_{6} A_{0}^{2} F_{\mu \nu}^{2}+c_{7} A_{0} F_{\mu \nu} A_{0} F_{\mu \nu}+c_{8} A_{0}^{2} F_{0 \mu}^{2}+c_{9} A_{0} F_{0 \mu} A_{0} F_{0 \mu}\right] \\
& \left.+g_{\mathrm{E}}^{4}\left[c_{10} A_{0}^{6}\right]\right\} .
\end{aligned}
$$

The colour trace refers to the adjoint representation: $\operatorname{tr}\{A B\} \equiv A_{a b} B_{b a}, \operatorname{tr}\{A B C\} \equiv$ $A_{a b} B_{b c} C_{c a}$, where $\left(A_{0}\right)_{a b} \equiv-i f^{a b c} A_{0}^{c}, \quad\left(F_{\mu 0}\right)_{a b} \equiv-i f^{a b c} F_{\mu 0}^{c}$, and $\left(D_{\mu} F_{\mu \nu}\right)_{a b} \equiv$ $-i f^{a b c} \mathcal{D}_{\mu}^{c d} F_{\mu \nu}^{d}$. The value of the sum-integral over $P$ evaluates to

$\oint_{P}^{\prime} \frac{1}{P^{6}}=\frac{\Gamma\left(3-\frac{d}{2}\right) \zeta(6-d) T}{(4 \pi)^{\frac{d}{2}}(2 \pi T)^{6-d}} \stackrel{3-2 \epsilon}{=} \frac{\zeta_{3} \mu^{-2 \epsilon}}{128 \pi^{4} T^{2}}\left\{1+2 \epsilon\left[\ln \left(\frac{\bar{\mu} e^{\gamma_{\mathrm{E}}}}{4 \pi T}\right)+1-\gamma_{\mathrm{E}}+\frac{\zeta_{3}^{\prime}}{\zeta_{3}}\right]+\mathcal{O}\left(\epsilon^{2}\right)\right\}$.

The values of $c_{i}$ were given for $d=3$ in ref. [28]. We need to generalize the expressions to $d$ dimensions, because some of the operators lead to divergent loop integrals at the second stage of our analysis (cf. section 3). Beyond leading order, the coefficients are also functions of $g^{2}$, but these contributions are of higher order than the effects that we are interested in. As mentioned in section 2.1, we are also suppressing effects proportional to $N_{\mathrm{f}}$.

As a first step, it may be realized that the operator basis in eq. (2.13) is redundant: it can be verified that

$$
\int_{X} \operatorname{tr}\left\{i g_{\mathrm{E}}\left[F_{0 \mu} F_{\mu \nu} F_{\nu 0}+A_{0}\left(D_{\mu} F_{\mu \nu}\right) F_{0 \nu}\right]+\frac{g_{\mathrm{E}}^{2}}{2}\left[-A_{0}^{2} F_{\mu \nu}^{2}+A_{0} F_{\mu \nu} A_{0} F_{\mu \nu}\right]\right\}=0 .
$$

Therefore a simultaneous change of the coefficients $\left(c_{i}^{\text {new }} \equiv c_{i}+\delta c_{i}, i=4, \ldots, 7\right)$ has no physical effect, provided that

$$
\delta c_{4}=\delta c_{5}=-2 \delta c_{6}=2 \delta c_{7}
$$

In particular, we could tune $c_{7}$ to zero as was done in ref. [28], ${ }^{2}$ by choosing $\delta c_{7}=-c_{7}$. Then eq. (2.16) implies that the other coefficients should appear in the combinations

$$
c_{4}^{\text {(new) }}=c_{4}-2 c_{7}, \quad c_{5}^{\text {(new) }}=c_{5}-2 c_{7}, \quad c_{6}^{\text {(new) }}=c_{6}+c_{7} .
$$

In the following we keep both $c_{5} \neq 0$ and $c_{7} \neq 0$ for generality; this offers for a good crosscheck in that only the combinations of eq. (2.17) appear in any physical expressions.

In order to determine the values of the coefficients $c_{i}$, we have computed 1-loop contributions to the 2-point, 3-point, 5-point and 6-point functions of the Matsubara zero modes in the background field Feynman gauge [26]. ${ }^{3}$ Salient details from this computation are

\footnotetext{
${ }^{2}$ Tuning $c_{5}$ to zero would yield eq. (2.13) more elegant and simplify a number of subsequent computations.

${ }^{3}$ In a general gauge, several of the coefficients depend on the gauge fixing parameter, but we have checked that the logarithmic divergences that we are ultimately interested in do not.
} 
presented in section 2.3. Matching the 2 and 3-point vertices yields

$$
c_{1}=\frac{41-d}{120}, \quad c_{2}=\frac{(d-1)(d-5)}{120}, \quad c_{3}=\frac{1-d}{180}, \quad c_{5}-c_{4}=\frac{(d-1)(d-5)}{60} .
$$

Adding the 5-point vertex permits for us to fix the combinations in eq. (2.17) as

$$
c_{4}-2 c_{7}=\frac{(41-d)(5-d)}{60}, \quad c_{5}-2 c_{7}=\frac{(21-d)(5-d)}{30}, \quad c_{6}+c_{7}=\frac{(d-25)(5-d)}{24} .
$$

In addition the 5-point vertex shows the presence of so-called evanescent operators whose coefficients vanish for $d=3$,

$$
c_{8}=\frac{(5-d)(3-d)(d-1)}{20}, \quad c_{9}=\frac{(5-d)(3-d)(d-1)}{30} .
$$

The coefficient $c_{10}$ is also evanescent and can be determined from the 6-point vertex; we find $c_{10}=(5-d)(3-d)(d-1)^{2} / 180$ but this does not contribute to any of our results. For $d=3$ eqs. (2.18)-(2.20) agree with ref. [28]. (Expressions for a general $d$ were derived in ref. [29], but unfortunately a rather different notation was employed.)

\subsection{Details on the determination of dimension-six coefficients}

In this section we provide some details on the determination of the coefficients listed in eqs. (2.18)-(2.20). The derivation of eq. (2.13) is most conveniently formulated with the background field method [26], and as a reminder the gauge potentials are denoted by $B_{\mu}^{a}$. The object computed is the background field effective action, $\Gamma_{\mathrm{EQCD}}[B]$, whereby the vertices are automatically symmetrized in the appropriate way. After a field redefinition, viz. $A_{i}^{a}=B_{i}^{a}\left(1+\mathcal{O}\left(g_{\mathrm{B}}^{2}\right)\right)$ and $A_{0}^{a}=B_{0}^{a}\left(1+\mathcal{O}\left(g_{\mathrm{B}}^{2}\right)\right)$, the result is identified with $S_{\mathrm{EQCD}}[A]$.

We choose to work directly in momentum space, with the background fields denoted by $B_{\mu}^{a}(q)$. The momenta $q$ have spatial components only:

$$
q_{\mu} \equiv \delta_{\mu i} q_{i}
$$

Specific tensors are defined for showing the dependence of the vertices on spacetime and colour indices; these are summarized in appendix A. The structure naturally emerging from the computation is one in which there are Lorentz-invariant structures $\left(\delta_{\mu \nu}\right.$ etc.) and additional terms that only appear for the zero components of the gauge potentials; the latter are identified through the tensors $T_{\mu \nu} \equiv \delta_{\mu 0} \delta_{\nu 0}$ etc. Results for various 1-loop sum-integrals in this basis are given in appendix B.

Computing the 2-point and 3-point vertices in the background field gauge, we obtain the 1-loop correction

$$
\begin{aligned}
\Gamma_{\mathrm{EQCD}}^{(2+3)}[B] & =\frac{g_{\mathrm{B}}^{2} N_{\mathrm{c}}}{2 !} B_{\mu}^{a}(q) B_{\nu}^{b}(r) \delta^{a b} \delta(q+r) \gamma_{\mu \nu}^{(2)}(q) \\
& +\frac{i g_{\mathrm{B}}^{3} N_{\mathrm{c}}}{3 !} B_{\mu}^{a}(q) B_{\nu}^{b}(r) B_{\rho}^{c}(s) f^{a b c} \delta(q+r+s) \gamma_{\mu \nu \rho}^{(3)}(q, r, s),
\end{aligned}
$$


where summations and integrations are implied, and $T \int_{q} \delta(q) \equiv 1$. Expanding in $1 / P^{2} \sim$ $1 /(\pi T)^{2}$, the 2 -point vertex reads

$$
\begin{gathered}
\gamma_{\mu \nu}^{(2)}(q)=\mathcal{F}_{P}^{\prime}\left\{\frac{(d-25)\left(q^{2} \delta_{\mu \nu}-q_{\mu} q_{\mu}\right)}{6 P^{4}}+T_{\mu \nu}\left[\frac{(d-1)^{2}}{P^{2}}-\frac{(d-1)(d-3) q^{2}}{6 P^{4}}\right]\right. \\
\left.+\frac{4 c_{1} q^{2}\left(q^{2} \delta_{\mu \nu}-q_{\mu} q_{\mu}\right)+4 c_{2} q^{4} T_{\mu \nu}}{P^{6}}+\mathcal{O}\left(\frac{1}{P^{8}}\right)\right\}
\end{gathered}
$$

where $c_{1}$ and $c_{2}$ have the values in eq. (2.18). The term proportional to $\mathcal{E}_{P}^{\prime} \frac{1}{P^{2}}$ yields the parameter $m_{\mathrm{E}}^{2}$ in eq. (2.2), whereas the terms proportional to $\mathcal{F}_{P}^{\prime} \frac{1}{P^{4}}$ yield wave function corrections. The existence of a term $\mathcal{E}_{P}^{\prime} \frac{T_{\mu \nu} q^{2}}{P^{4}}$ indicates that temporal and spatial components of the gauge potentials need to be normalized differently.

For the 3-point vertex a similar computation leads to

$$
\begin{aligned}
\gamma_{\mu \nu \rho}^{(3)}(q, r, s) & \mathcal{F}_{P}^{\prime}\left\{\frac{(25-d) q_{\rho} \delta_{\mu \nu}+(d-1)(d-3) q_{\rho} T_{\mu \nu}}{P^{4}}\right. \\
& -\frac{24 c_{1} q_{\mu} q_{\rho} r_{\nu}+12 c_{3} q_{\nu}\left(r_{\mu} q_{\rho}-q_{\mu} r_{\rho}\right)}{P^{6}} \\
& -\frac{6\left(4 c_{1}-3 c_{3}\right) s^{2} q_{\rho} \delta_{\mu \nu}-6 q^{2}\left[3 c_{3} s_{\rho}+8 c_{1} r_{\rho}\right] \delta_{\mu \nu}}{P^{6}} \\
& \left.+\frac{6\left(c_{4}-c_{5}\right) s^{2} q_{\rho} T_{\mu \nu}-6 q^{2}\left[4 c_{2}\left(q_{\rho}-r_{\rho}\right)+\left(c_{5}-c_{4}\right) s_{\rho}\right] T_{\mu \nu}}{P^{6}}+\mathcal{O}\left(\frac{1}{P^{8}}\right)\right\},
\end{aligned}
$$

where $c_{3}$ and $c_{4}-c_{5}$ have the values shown in eq. (2.18). ${ }^{4}$ The terms proportional to $\mathbb{f}_{P}^{\prime} \frac{1}{P^{4}}$ can be partly accounted for by wave function corrections; the remainder yields the effective gauge coupling of eq. (2.3). The same result for $g_{\mathrm{E}}^{2}$ is obtained both from a purely spatial vertex $\left(\sim q_{\rho} \delta_{\mu i} \delta_{\nu i}\right)$ and from a vertex mixing two $A_{0}^{a}$ 's and one $A_{i}^{a}\left(\sim q_{\rho} T_{\mu \nu}\right)$.

The 4-point vertex can similarly be written as

$$
\Gamma_{\mathrm{EQCD}}^{(4)}[B]=\frac{g_{\mathrm{B}}^{4}}{4 !} B_{\mu}^{a}(q) B_{\nu}^{b}(r) B_{\alpha}^{c}(s) B_{\beta}^{d}(t) \delta(q+r+s+t) \gamma_{\mu \nu \alpha \beta}^{(4) a b c d}(q, r, s, t),
$$

where

$$
\begin{aligned}
\gamma_{\mu \nu \alpha \beta}^{(4) a b c d}(q, r, s, t) & =\mathcal{f}_{P}^{\prime}\left\{X^{\{a b\}\{c d\}} \frac{2(d-1)^{2}(3-d) T_{\mu \nu \alpha \beta}}{P^{4}}\right. \\
& \left.+X^{[a b][c d]} \frac{4(25-d) \delta_{\mu \alpha} \delta_{\nu \beta}+8(d-1)(d-3) T_{\mu \alpha} \delta_{\nu \beta}}{P^{4}}+\mathcal{O}\left(\frac{1}{P^{6}}\right)\right\} .
\end{aligned}
$$

The notations $X^{\{a b\}\{c d\}}$ and $X^{[a b][c d]}$ are defined in appendix A. The term proportional to $\mathbb{E}_{P}^{\prime} \frac{T_{\mu \nu \alpha \beta}}{P^{4}}$ yields $\lambda_{\mathrm{E}}$ in eq. (2.4), whereas the other terms proportional to $\mathfrak{K}_{P}^{\prime} \frac{1}{P^{4}}$ correspond to wave function corrections and $g_{\mathrm{E}}^{2}$. The dimension-six part of the 4-point vertex is rather complicated (it is shown in appendix $\mathrm{C}$ ) and we have not used it for determining $c_{i}$ 's.

\footnotetext{
${ }^{4}$ This representation is not unique, cf. the comments below eq. (C.3).
} 

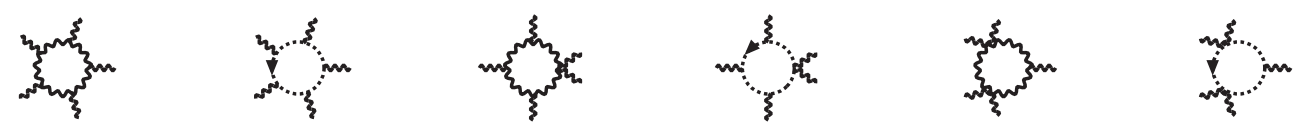

Figure 1. 1-loop contributions to the 5-point function in the background field gauge. Wiggly lines denote gluons and dotted lines ghosts. The diagrams have been drawn with Axodraw [30].

Proceeding finally to the 5-point vertex, we find no contribution $\sim \mathfrak{f}_{P}^{\prime} \frac{1}{P^{4}}$. The contribution of the dimension-six operators from eq. (2.13) can be written as

$$
\begin{aligned}
\Gamma_{\mathrm{EQCD}}^{(5)}[B]=B_{\mu}^{a}(q) B_{\nu}^{b}(r) & B_{\rho}^{c}(s) B_{\alpha}^{d}(t) B_{\beta}^{e}(u) \delta(q+r+s+t+u)\left(\oint_{P}^{\prime} \frac{8 i g_{\mathrm{E}}^{5} s_{\mu}}{P^{6}}\right) \\
\times\left\{X^{\{a b\}[c d e]}[\right. & -c_{1} \delta_{\rho \alpha} \delta_{\nu \beta}+4 c_{1} \delta_{\rho \beta} \delta_{\nu \alpha}-c_{1} \delta_{\rho \nu} \delta_{\alpha \beta} \\
& -c_{2} T_{\rho \alpha} \delta_{\nu \beta}+4 c_{2} T_{\rho \beta} \delta_{\nu \alpha}-c_{2} T_{\rho \nu} \delta_{\alpha \beta} \\
& \left.-c_{2} \delta_{\rho \alpha} T_{\nu \beta}+\left(c_{5}-2 c_{7}\right) \delta_{\rho \beta} T_{\nu \alpha}-c_{2} \delta_{\rho \nu} T_{\alpha \beta}-c_{9} T_{\rho \nu \alpha \beta}\right] \\
+X^{[a b]\{c d e\}} & {\left[\left(5 c_{1}-3 c_{3}\right) \delta_{\rho \alpha} \delta_{\nu \beta}+\left(3 c_{3}-4 c_{1}\right) \delta_{\rho \beta} \delta_{\nu \alpha}+3 c_{1} \delta_{\rho \nu} \delta_{\alpha \beta}\right.} \\
+ & \left(c_{2}-c_{4}+c_{5}\right) T_{\rho \alpha} \delta_{\nu \beta}+\left(c_{4}-c_{5}\right) T_{\rho \beta} \delta_{\nu \alpha}+3 c_{2} T_{\rho \nu} \delta_{\alpha \beta} \\
+ & \left(c_{2}-c_{4}+c_{5}\right) \delta_{\rho \alpha} T_{\nu \beta}+\left(c_{4}-4 c_{2}-2 c_{7}\right) \delta_{\rho \beta} T_{\nu \alpha} \\
+ & \left.\left.\left(c_{5}-c_{2}+2 c_{6}\right) \delta_{\rho \nu} T_{\alpha \beta}+\left(c_{8}-c_{9}\right) T_{\rho \nu \alpha \beta}\right]\right\}+\mathcal{O}\left(\mathcal{F}_{P}^{\prime} \frac{1}{P^{8}}\right) .
\end{aligned}
$$

We have computed the corresponding Feynman diagrams, shown in figure 1. Making use of momentum conservation and appropriate symmetrizations, and identifying $g_{\mathrm{E}}^{2}=$ $g_{\mathrm{B}}^{2}\left(1+\mathcal{O}\left(g_{\mathrm{B}}^{2}\right)\right)$, we obtain precisely the same structure from Feynman diagrams. There are 20 independent terms that permit for a crosscheck of eq. (2.18) and, most importantly, for a unique determination of the combinations appearing in eqs. (2.19) and (2.20).

\section{Overlapping soft/hard and ultrasoft/hard contributions}

In EQCD, the gauge field components $A_{0}^{a}$ have turned into massive adjoint scalar fields when the non-zero Matsubara modes were integrated out (cf. eq. (2.1)). Our goal now is to integrate out the massive $A_{0}^{a}$, and thereby construct the MQCD action. Its superrenormalizable part has the form of the spatial part of eq. (2.1). We denote it by

$$
S_{\mathrm{MQCD}}[A] \equiv \int_{X} \frac{1}{4} F_{i j}^{a} F_{i j}^{a}
$$

even though $F_{i j}^{a}$ now contains a different gauge coupling than eq. (2.1): $F_{i j}^{a}=\partial_{i} A_{j}^{a}-$ $\partial_{j} A_{i}^{a}+g_{\mathrm{M}} f^{a b c} A_{i}^{b} A_{j}^{c}$. The main goal of this section is to determine the contributions to $g_{\mathrm{M}}^{2}$ that originate from the dimension-six operators in eq. (2.13). These are termed soft/hard (sections 3.1 and 3.2) and ultrasoft/hard (section 3.3) contributions.

We note that in analogy with eq. (2.13), $S_{\mathrm{MQCD}}$ also has a dimension-six part, $\delta S_{\mathrm{MQCD}}$. It is given in eq. (3.16) and discussed in more detail in section 3.3. 


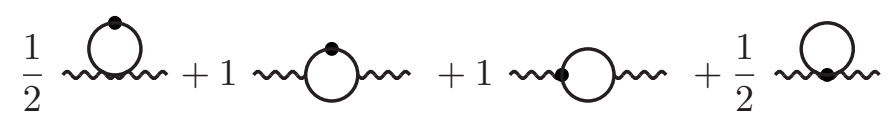

Figure 2. 1-loop contributions to the 2-point function, containing some of the "Chapman vertices" from eq. (2.13), denoted by a filled blob. The adjoint scalar fields are denoted by solid lines.

In order to determine $g_{\mathrm{M}}^{2}$, we once again make use of the background field effective action, $\Gamma_{\mathrm{MQCD}}[B]$. In particular, we consider its quadratic part,

$$
\Gamma_{\mathrm{MQCD}}^{(2)}[B]=\frac{1}{2} B_{i}^{a}(q) B_{j}^{a}(-q)\left(q^{2} \delta_{i j}-q_{i} q_{j}\right)\left(Z_{B}+\delta Z_{B}\right),
$$

where $\delta Z_{B}$ collects any possible divergences.

In the background field gauge, $\Gamma$ is gauge invariant in terms of $B$ [26]. Consequently the 3-point and 4-point vertices are fully determined by eq. (3.2). After a subsequent field redefinition, this implies that $Z_{B}$ determines the gauge coupling of MQCD:

$$
g_{\mathrm{M}}^{2}=g_{\mathrm{ER}}^{2} \mu^{2 \epsilon} Z_{B}^{-1}-g_{\mathrm{ER}}^{2} \mu^{2 \epsilon} \delta Z_{B}+\delta g_{\mathrm{E}}^{2}+\mathcal{O}\left(g^{10}\right)
$$

Here $\delta g_{\mathrm{E}}^{2}$ is from eq. (2.8). The following discussion is carried out in terms of $Z_{B}$ and $\delta Z_{B}$.

When the field $A_{0}^{a}$ is integrated out and one vertex from eq. (2.13) is included, we expect to find terms of the types

$$
Z_{B}+\delta Z_{B}=1+\left(\oiint_{P}^{\prime} \frac{g_{\mathrm{E}}^{2} N_{\mathrm{c}}}{P^{6}}\right)\left[\frac{m_{\mathrm{ER}} g_{\mathrm{ER}}^{2} N_{\mathrm{c}} T}{4 \pi} \#^{(5)}+\frac{\left(g_{\mathrm{ER}}^{2} N_{\mathrm{c}} T\right)^{2}}{(4 \pi)^{2}} \#^{(6)}+\ldots\right],
$$

where $\#^{(6)}$ may contain logarithms. The corresponding effects are of $\mathcal{O}\left(g^{5}\right)$ and $\mathcal{O}\left(g^{6}\right)$ in terms of the original QCD coupling. The latter effect is comparable to eq. (2.12).

Before proceeding let us explain why we consider "2-loop soft $\times 1$-loop hard" contributions, i.e. 2-loop graphs with one insertion of dimension-six operators, but not "1-loop soft $\times$ 2-loop hard" ones. In terms of $Z_{B}$ defined in eq. (3.2), "1-loop hard" gives a factor $\sim g^{2} / T^{2}$, "1-loop soft" gives a factor $\sim g^{2} T m_{\mathrm{ER}} \sim g^{3} T^{2}$, and "2-loop soft" gives a factor $\sim\left(g^{2} T\right)^{2} \sim g^{4} T^{2}$. The overall effects of these orders are $\sim g^{5}, g^{6}$, cf. eq. (3.4). In contrast "2-loop hard" would give dimension-six operators proportional to $\sim g^{4} / T^{2}$. The overall effect from "1-loop soft $\times 2$-loop hard" would therefore be $\sim g^{7}$, i.e. of higher order than our computation. The same applies to dimension-eight operators, whose coefficients are $\sim g^{2} / T^{4}$ and who get a further suppression factor $\lesssim g^{2} T m_{\mathrm{ER}}^{3} \sim g^{5} T^{4}$ from soft effects.

\subsection{1-loop results with dimension-six operators}

The 1-loop contribution to $Z_{B}$ from dimension-six operators originates from the graphs shown in figure 2 . The vertices related to dimension-six operators have been indicated with a filled blob; we refer to them as "Chapman vertices". In appendix C the vertices are written in a form convenient for computing these graphs. The 2-point vertex is parametrized through $\eta_{1}, \eta_{2}$, cf. eq. (C.1); the 3-point vertex through $\xi_{1}, \ldots, \xi_{10}$, cf. eq. (C.3); and the 4-point vertex through $\psi_{1}, \ldots, \psi_{44}$ and $\omega_{1}, \ldots, \omega_{35}$, cf. eq. (C.5). 
Computing the graphs in figure 2 in dimensional regularization and expanding in $q^{2} / m_{\mathrm{E}}^{2}$, all of them can be related to a single 1-loop tadpole integral, denoted by

$$
I\left(m_{\mathrm{E}}\right) \equiv \int_{p} \frac{T}{p^{2}+m_{\mathrm{E}}^{2}}=\frac{m_{\mathrm{E}}^{d-2} \Gamma\left(1-\frac{d}{2}\right) T}{(4 \pi)^{\frac{d}{2}}} \stackrel{3-2 \epsilon}{=}-\frac{m_{\mathrm{E}} T \mu^{-2 \epsilon}}{4 \pi}\left[1+2 \epsilon\left(1+\ln \frac{\bar{\mu}}{2 m_{\mathrm{E}}}\right)+\mathcal{O}\left(\epsilon^{2}\right)\right] .
$$

We get

$$
\begin{aligned}
\delta \Gamma_{\mathrm{MQCD}}^{(2)}[B]= & B_{i}^{a}(q) B_{j}^{b}(r) \delta^{a b} \delta(q+r)\left(\xi_{P}^{\prime} \frac{g_{\mathrm{E}}^{4} N_{\mathrm{c}}^{2}}{P^{6}}\right) I\left(m_{\mathrm{E}}\right) \\
& \times\left\{m _ { \mathrm { E } } ^ { 2 } \delta _ { i j } \left[\frac{d+2}{d}\left(-2 \eta_{2}-\xi_{8}+\xi_{9}\right)-\frac{3}{4}\left(\psi_{4}+\frac{\psi_{26}}{d}\right)\right.\right. \\
& \left.\quad-\psi_{13}+\psi_{15}+\frac{1}{d}\left(\psi_{35}-\psi_{34}\right)+\frac{1}{4}\left(\omega_{4}+\frac{\omega_{26}}{d}\right)\right] \\
+ & +\left(q^{2} \delta_{i j}-q_{i} q_{j}\right)\left[\frac{(4+d)(2-d)}{24} \eta_{2}+\frac{d-2}{12}\left(\xi_{9}-\xi_{8}\right)+\xi_{10}\right. \\
& \left.+\frac{3 \psi_{5}}{4}+\psi_{16}-\psi_{18}-\frac{\omega_{5}}{4}\right] \\
+ & q_{i} q_{j}\left[\eta_{2}+\xi_{8}+\xi_{10}+\frac{3}{4}\left(\psi_{5}+\psi_{29}\right)+\psi_{16}-\psi_{18}\right. \\
& \left.\left.+\psi_{42}-\psi_{43}-\psi_{44}-\frac{1}{4}\left(\omega_{5}+\omega_{29}\right)\right]+\mathcal{O}\left(\frac{q^{4}}{m_{\mathrm{E}}^{2}}\right)\right\} .
\end{aligned}
$$

Inserting the values of the coefficients in terms of the $c_{i}$ 's from appendix $\mathrm{C}$, the terms proportional to $m_{\mathrm{E}}^{2} \delta_{i j}$ and $q_{i} q_{j}$ drop out as required by gauge invariance, and we are left with

$$
\begin{aligned}
\delta \Gamma_{\mathrm{MQCD}}^{(2)}[B]= & B_{i}^{a}(q) B_{j}^{b}(r) \delta^{a b} \delta(q+r)\left(q^{2} \delta_{i j}-q_{i} q_{j}\right)\left(\sum_{P}^{\prime} \frac{g_{\mathrm{E}}^{4} N_{\mathrm{c}}^{2}}{P^{6}}\right) I\left(m_{\mathrm{E}}\right) \\
& \times\left\{\frac{(4-d)(d-2)}{12}\left(c_{1}+c_{2}\right)+3 c_{3}+\left(c_{4}-2 c_{7}\right)+4\left(c_{6}+c_{7}\right)\right\} .
\end{aligned}
$$

Inserting the coefficients $c_{1}, \ldots, c_{7}$ from eqs. (2.18) and (2.19) and setting $d \rightarrow 3$, the curly brackets evaluate to

$$
\lim _{d \rightarrow 3}\{\ldots\}=-\lim _{d \rightarrow 3} \frac{d^{4}-13 d^{3}+312 d^{2}-6404 d+25424}{1440}=-\frac{875}{144} .
$$

The corresponding contribution to $Z_{B}$ is shown on the first row of eq. (3.13).

\section{$3.2 \quad 2$-loop results with dimension-six operators}

At 2-loop level, the contributions of the 2-point, 3-point and 4-point Chapman vertices to $Z_{B}$ can be extracted from Feynman diagrams shown in figures $3-5$. In addition the 5-point and 6-point Chapman vertex also contribute. The general expressions for these, parametrized through the coefficients $\kappa_{1}, \ldots, \kappa_{10}, \lambda_{1}, \ldots, \lambda_{10}$ and $\chi_{1}, \ldots, \chi_{16}$, are given in eqs. (C.19) and (C.21), respectively, and the corresponding diagrams are shown in figure 6. 


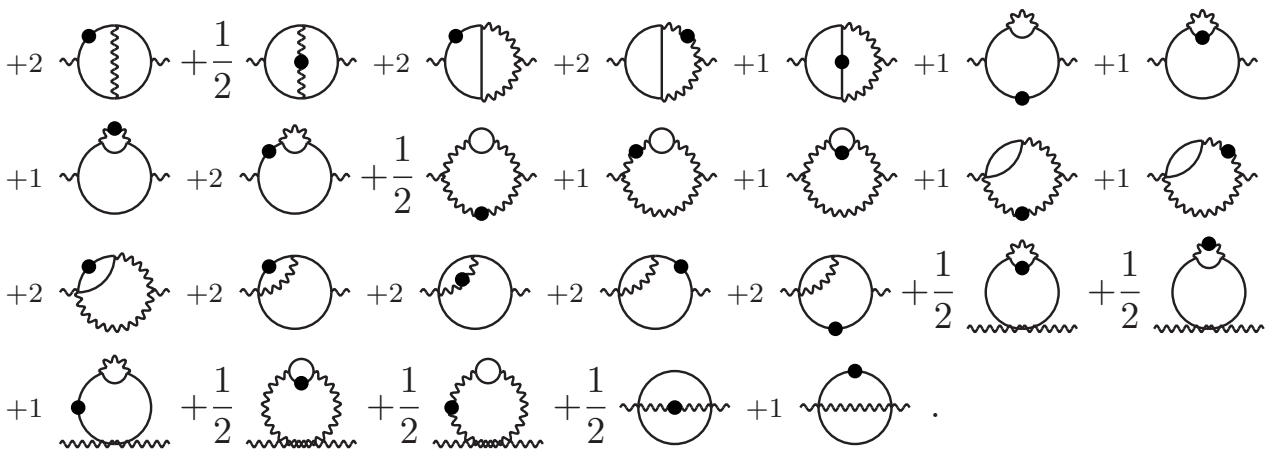

Figure 3. 2-loop contributions to the 2-point function, originating from 2-point Chapman vertices, denoted by filled blobs. Adjoint scalars are denoted by solid lines. Graphs involving closed massless loops, which do not contribute to the matching, have been omitted.

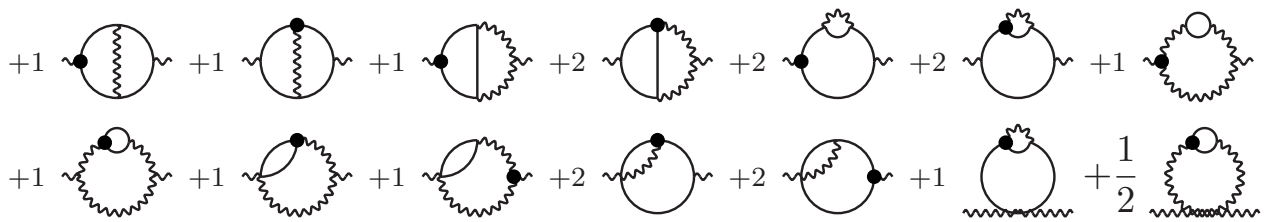

Figure 4. 2-loop contributions to the 2-point function, originating from 3-point Chapman vertices (the notation is as in figure 3).

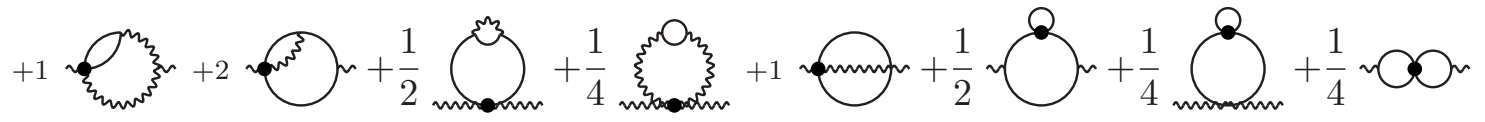

Figure 5. 2-loop contributions to the 2-point function, originating from 4-point Chapman vertices (the notation is as in figure 3 ).

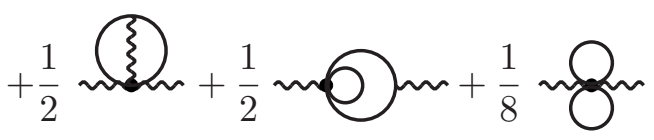

Figure 6. 2-loop contributions to the 2-point function, originating from 5-point or 6-point Chapman vertices (the notation is as in figure 3 ).

In order to display the result, we introduce a 2-loop "sunset" integral,

$$
\begin{aligned}
H\left(m_{\mathrm{E}}\right) & \equiv \int_{p, q} \frac{T^{2}}{\left(p^{2}+m_{\mathrm{E}}^{2}\right)\left(q^{2}+m_{\mathrm{E}}^{2}\right)(p+q)^{2}} \\
& =\frac{m_{\mathrm{E}}^{2 d-6} \Gamma\left(1-\frac{d}{2}\right) \Gamma\left(2-\frac{d}{2}\right) T^{2}}{(d-3)(4 \pi)^{d}} \stackrel{3-2 \epsilon}{=} \frac{T^{2} \mu^{-4 \epsilon}}{(4 \pi)^{2}}\left[\frac{1}{4 \epsilon}+\ln \left(\frac{\bar{\mu}}{2 m_{\mathrm{E}}}\right)+\frac{1}{2}+\mathcal{O}(\epsilon)\right] .
\end{aligned}
$$


Then

$$
\begin{aligned}
\delta \Gamma_{\mathrm{MQCD}}^{(2)}[B]= & \frac{1}{2} B_{i}^{a}(q) B_{j}^{b}(r) \delta^{a b} \delta(q+r)\left(\sum_{P}^{\prime} \frac{g_{\mathrm{E}}^{6} N_{\mathrm{c}}^{3}}{P^{6}}\right) H\left(m_{\mathrm{E}}\right) \\
& \times\left\{\frac{m_{\mathrm{E}}^{2} \delta_{i j}}{4 d} C_{1}+\frac{q^{2} \delta_{i j}-q_{i} q_{j}}{4 d} C_{2}+\frac{q_{i} q_{j}}{4 d} C_{3}+\mathcal{O}\left(\frac{q^{4}}{m_{\mathrm{E}}^{2}}\right)\right\},
\end{aligned}
$$

where $C_{1}, C_{2}, C_{3}$ are given in appendix $\mathrm{E}$ in terms of the coefficients $\eta_{1}, \ldots, \chi_{16} \cdot{ }^{5}$

Inserting the values of the coefficients from appendix $\mathrm{C}$, we find that $C_{1}$ and $C_{3}$ and terms proportional to $\alpha$ in $C_{2}$ cancel. The remaining contribution reads

$$
\begin{aligned}
\delta \Gamma_{\mathrm{MQCD}}^{(2)}[B]= & -B_{i}^{a}(q) B_{j}^{b}(r) \delta^{a b} \delta(q+r)\left(q^{2} \delta_{i j}-q_{i} q_{j}\right)\left(\sum_{P}^{\prime} \frac{g_{\mathrm{E}}^{6} N_{\mathrm{c}}^{3}}{P^{6}}\right) H\left(m_{\mathrm{E}}\right) \\
& \times\left\{\frac{(d-3)(d-4)^{2}\left(d^{3}-10 d^{2}+23 d-44\right)\left(c_{1}+c_{2}\right)}{6 d(d-5)(d-7)}\right. \\
& +\frac{\left(d^{4}-18 d^{3}+95 d^{2}-210 d+192\right) c_{3}}{2 d(d-5)}+\frac{\left(d^{3}-13 d^{2}+36 d-36\right)\left(c_{4}-2 c_{7}\right)}{6 d} \\
& \left.+\frac{2\left(d^{3}-13 d^{2}+21 d-6\right)\left(c_{6}+c_{7}\right)}{3 d}+\frac{(d-3)(d-4)\left(2 c_{8}+c_{9}\right)}{6}\right\} \\
= & -B_{i}^{a}(q) B_{j}^{b}(r) \delta^{a b} \delta(q+r)\left(q^{2} \delta_{i j}-q_{i} q_{j}\right)\left(\sum_{P}^{\prime} \frac{g_{\mathrm{E}}^{6} N_{\mathrm{c}}^{3}}{P^{6}}\right) H\left(m_{\mathrm{E}}\right) \\
& \times\left(17 d^{8}-494 d^{7}+6522 d^{6}-53766 d^{5}+301049 d^{4}-1075772 d^{3}\right. \\
& \left.+2085956 d^{2}-1575176 d+102864\right) \frac{1}{720 d(d-5)(d-7)}
\end{aligned}
$$

where in the last step we made use of eqs. (2.18)-(2.20). We note that the evanescent operators parametrized by $c_{8}$ and $c_{9}$ do not play a role for $d \approx 3$, because the coefficients with which they contribute in eq. (3.11) themselves vanish for $d \rightarrow 3$.

Setting $d=3-2 \epsilon$, inserting eqs. (2.14), (3.7) and (3.9), and going over to renormalized parameters, we obtain

$$
\begin{aligned}
Z_{B}= & +\left(\frac{g_{\mathrm{ER}}^{2} N_{\mathrm{c}}}{16 \pi^{2}}\right)^{2} \frac{m_{\mathrm{ER}}}{2 \pi T}\left(\frac{875 \zeta_{3}}{72}\right) \\
& -\left(\frac{g_{\mathrm{ER}}^{2} N_{\mathrm{c}}}{16 \pi^{2}}\right)^{3}\left(\frac{1097 \zeta_{3}}{549}\right) \frac{61}{5}\left\{\ln \left(\frac{\bar{\mu} e^{\gamma_{\mathrm{E}}}}{4 \pi T}\right)+2 \ln \left(\frac{\bar{\mu}}{2 m_{\mathrm{ER}}}\right)+\frac{\zeta_{3}^{\prime}}{\zeta_{3}}-\gamma_{\mathrm{E}}+\frac{103771}{52656}\right\} \\
\delta Z_{B}= & -\left(\frac{g_{\mathrm{ER}}^{2} N_{\mathrm{c}}}{16 \pi^{2}}\right)^{3}\left(\frac{1097 \zeta_{3}}{1098}\right) \frac{61}{5 \epsilon} .
\end{aligned}
$$

Remarkably, setting $g_{\mathrm{ER}}^{2}=g^{2}\left(1+\mathcal{O}\left(g^{2}\right)\right)$, the divergence in eq. (3.14) cancels $1097 / 1098$ of the coefficient of $1 / \epsilon$ in eq. (2.12). The remaining $1 / 1098$ can be expressed as

$$
\delta \mathcal{Z}_{B}+\delta Z_{B}=\frac{g^{6} N_{\mathrm{c}}^{3} T^{2}}{(8 \pi)^{2}}\left(\frac{\zeta_{3}}{128 \pi^{4} T^{2}}\right) \frac{1}{45 \epsilon}+\mathcal{O}\left(g^{8}\right)
$$

where in the round brackets we have isolated the master integral in eq. (2.14).

${ }^{5} \mathrm{~A}$ general gauge parameter, denoted by $\alpha$, has been employed: $\left\langle A_{k}^{a}(p) A_{l}^{b}(q)\right\rangle \equiv \frac{\delta^{a b} \delta(p+q)}{p^{2}}\left(\delta_{k l}-\frac{\alpha p_{k} p_{l}}{p^{2}}\right)$. 


\subsection{Contribution from dimension-six operators in MQCD}

As already alluded to below eq. (3.1), there are dimension-six operators also in MQCD. These originate from the purely spatial part of eq. (2.13), and also from 1-loop effects within EQCD, as will be discussed in section 4 . The corresponding action can be written $\operatorname{as}^{6}$

$$
\delta S_{\mathrm{MQCD}}[A]=2 g_{\mathrm{M}}^{2} \int_{X} \operatorname{tr}\left\{\mathcal{C}_{1}\left(D_{i} F_{i j}\right)^{2}+i g_{\mathrm{M}} \mathcal{C}_{3} F_{i j} F_{j k} F_{k i}\right\},
$$

where (recalling $\left.g_{\mathrm{M}}^{2}=g_{\mathrm{E}}^{2}(1+\mathcal{O}(g))\right)$ the hard contribution is $\delta \mathcal{C}_{i}=\mathcal{F}_{P}^{\prime} c_{i} / P^{6}$.

The dimension-six operators in eq. (3.16) give a contribution to physical observables determined by MQCD, such as the spatial string tension or "magnetostatic" screening masses. Given that MQCD is a confining theory, these effects cannot be computed analytically. We would like to know, however, whether the MQCD dynamics can give an ultraviolet (UV) divergent contribution, compensating against the term in eq. (3.15).

In order to determine the UV divergence, we employ a trick similar to that in ref. [31]. All infrared (IR) contributions are "shielded" by employing the propagators

$$
\left\langle A_{k}^{a}(p) A_{l}^{b}(q)\right\rangle \equiv \frac{\delta^{a b} \delta(p+q)}{p^{2}+m_{\mathrm{G}}^{2}}\left(\delta_{k l}-\frac{\alpha p_{k} p_{l}}{p^{2}+m_{\mathrm{G}}^{2}}\right), \quad\left\langle c^{a}(p) \bar{c}^{b}(q)\right\rangle \equiv \frac{\delta^{a b} \delta(p-q)}{p^{2}+m_{\mathrm{G}}^{2}},
$$

where $c^{a}, \bar{c}^{b}$ are ghost fields, $\alpha$ is a gauge parameter, and $m_{\mathrm{G}} \equiv g_{\mathrm{M}}^{2} T / \pi$ is a fictitious mass. Once again, we compute a background field effective action, now denoted by $\Gamma_{\mathrm{IR}}[B]$ given that the most IR fluctuations have been accounted for. We extract from it a 2-point function like in eq. (3.2). The technical implementation follows that in sections 3.1 and 3.2.

Most contributions that we find are $\alpha$-dependent and void of physical significance. For instance, the 1-loop result has a structure similar to eq. (3.6) but with $m_{\mathrm{E}} \rightarrow m_{\mathrm{G}}$ :

$$
\begin{aligned}
\left.\delta \Gamma_{\mathrm{IR}}^{(2)}[B]\right|_{\alpha=0}= & \frac{1}{2} B_{i}^{a}(q) B_{j}^{b}(r) \delta^{a b} \delta(q+r) g_{\mathrm{M}}^{4} N_{\mathrm{c}}^{2} I\left(m_{\mathrm{G}}\right) \\
& \times\left\{\left(q^{2} \delta_{i j}-q_{i} q_{j}\right)\left[-\frac{11 \mathcal{C}_{1}}{3}+18 \mathcal{C}_{3}+\mathcal{O}(\epsilon)\right]+\mathcal{O}\left(\frac{q^{4}}{m_{\mathrm{G}}^{2}}\right)\right\} .
\end{aligned}
$$

This result is finite and proportional to $m_{\mathrm{G}}$ and vanishes when we send $m_{\mathrm{G}} \rightarrow 0$.

However, at 2-loop order a non-trivial and gauge-independent result emerges. Writing the contribution from Chapman vertices in a form reminiscent of eq. (3.10), we get

$$
\begin{aligned}
\delta \Gamma_{\mathrm{IR}}^{(2)}[B]= & \frac{1}{2} B_{i}^{a}(q) B_{j}^{b}(r) \delta^{a b} \delta(q+r) g_{\mathrm{M}}^{6} N_{\mathrm{c}}^{3} H_{3}\left(m_{\mathrm{G}}\right) \\
& \times\left\{\frac{m_{\mathrm{G}}^{2} \delta_{i j}}{4 d} D_{1}+\frac{q^{2} \delta_{i j}-q_{i} q_{j}}{4 d} D_{2}+\frac{q_{i} q_{j}}{4 d} D_{3}+\mathcal{O}\left(\frac{q^{4}}{m_{\mathrm{G}}^{2}}\right)\right\} .
\end{aligned}
$$

The function $\mathrm{H}_{3}$ is the three-mass variant of eq. (3.9), cf. eq. (D.10), and has the same UV divergence, viz. $T^{2} \mu^{-4 \epsilon} /\left[(4 \pi)^{2} 4 \epsilon\right]$. The coefficients $D_{i}$ contain a part $\propto H / H_{3}=1+\mathcal{O}(\epsilon)$.

\footnotetext{
${ }^{6}$ There are many alternative representations, for instance $\operatorname{tr}\left\{F_{i j} F_{j k} F_{k i}\right\}=\frac{i N_{c}}{2} f^{a b c} F_{i j}^{a} F_{j k}^{b} F_{k i}^{c}=$ $\frac{i N_{c}}{2(d-2)} f^{a b c} \epsilon_{i j k} \widetilde{F}_{i}^{a} F_{j l}^{b} F_{k l}^{c}=\frac{i N_{c}}{2} f^{a b c} \widetilde{F}_{i}^{a} \widetilde{F}_{j}^{b} F_{i j}^{c}=\frac{i N_{c}}{2(d-2)} f^{a b c} \epsilon_{i j k} \widetilde{F}_{i}^{a} \widetilde{F}_{j}^{b} \widetilde{F}_{k}^{c}$, where we denoted the dual field strength by $\widetilde{F}_{i}^{a} \equiv \frac{\epsilon_{i j k}}{2} F_{j k}^{a}$ and defined $\epsilon_{i j k} \epsilon_{l m n} \equiv \delta_{i l}\left(\delta_{j m} \delta_{k n}-\delta_{j n} \delta_{k m}\right)+\delta_{i m}\left(\delta_{j n} \delta_{k l}-\delta_{j l} \delta_{k n}\right)+\delta_{i n}\left(\delta_{j l} \delta_{k m}-\right.$ $\left.\delta_{j m} \delta_{k l}\right)$.
} 
For $\epsilon \rightarrow 0, D_{1,3}$ are of $\mathcal{O}(\epsilon)$ and yield no divergence, whereas $D_{2}$ has a finite $\alpha$-independent part:

$$
D_{2}=24 \mathcal{C}_{3}+\mathcal{O}(\epsilon)
$$

Substituting $\mathcal{C}_{3} \rightarrow \mathcal{E}_{P}^{\prime} c_{3} / P^{6}$, inserting $c_{3}$ from eq. (2.18), and setting $g_{\mathrm{M}}^{2}=g^{2} \mu^{2 \epsilon}(1+$ $\mathcal{O}(g)$ ), yields a gauge-independent UV divergence and logarithmic part:

$$
\begin{aligned}
\delta \Gamma_{\mathrm{IR}}^{(2)}[B]= & \frac{1}{2} B_{i}^{a}(q) B_{j}^{b}(r) \delta^{a b} \delta(q+r)\left(q^{2} \delta_{i j}-q_{i} q_{j}\right) \\
& \times \frac{g^{6} N_{\mathrm{c}}^{3} T^{2}}{(8 \pi)^{2}}\left(\frac{\zeta_{3}}{128 \pi^{4} T^{2}}\right)\left(-\frac{1}{45}\right)\left\{\frac{1}{\epsilon}+2 \ln \left(\frac{\bar{\mu} e^{\gamma_{\mathrm{E}}}}{4 \pi T}\right)+4 \ln \left(\frac{\bar{\mu}}{3 m_{\mathrm{G}}}\right)+\mathcal{O}(1)\right\} .
\end{aligned}
$$

Comparing with eq. (3.15), the divergence exactly cancels. Therefore we have now established our main technical goal, demonstrating that the IR-divergence in eq. (2.12) is fully cancelled by soft/hard and ultrasoft/hard contributions from dimension-six operators.

\section{Soft and overlapping ultrasoft/soft contributions}

In section 3 we considered the soft/hard contributions to the MQCD effective action, cf. eq. (3.4). However, there are other contributions to $Z_{B}$, namely those associated with the purely "soft" contributions from the scale $m_{\mathrm{E}}$. In order to distinguish these from the effects considered in section 3 , we denote them by $\widetilde{Z}_{B}$. For this section, we can take the super-renormalizable truncation in eq. (2.1) as a starting point, and $m_{\mathrm{E}}$ as the only scale being integrated out.

\subsection{Direct soft terms up to 3-loop level}

Up to 2-loop level, the value of $\widetilde{Z}_{B}$ was determined in ref. [32] (the dependence on scalar couplings was added in ref. [20]): ${ }^{7}$

$$
\widetilde{Z}_{B}=1+\frac{g_{\mathrm{ER}}^{2} N_{\mathrm{c}} T}{48 \pi m_{\mathrm{ER}}}+\left(\frac{g_{\mathrm{ER}}^{2} N_{\mathrm{c}} T}{16 \pi m_{\mathrm{ER}}}\right)^{2}\left(\frac{19}{18}+\frac{4 \lambda}{3}\right)+\mathcal{O}\left(\frac{g_{\mathrm{ER}}^{2} N_{\mathrm{c}} T}{16 \pi m_{\mathrm{ER}}}\right)^{3} .
$$

We now turn to the 3-loop contribution.

The determination of $\widetilde{Z}_{B}$ is a rather straightforward exercise in computer-algebraic methods for loop integrals. The Feynman diagrams were generated with QGRAF [33]. After expanding in the external momentum and projecting onto the transverse and longitudinal polarizations, we have to deal with vacuum-like master integrals. The subsequent simplifications, making use of renamings of integration variables and integration-by-parts (IBP) identities [34, 35], have been programmed in FORM [36]. The values of the 3-loop master integrals can be found in refs. [31, 37] and are given in eqs. (D.12) and (D.13). As a crosscheck, we have carried out two independent computations, whose results coincide

\footnotetext{
${ }^{7}$ In $d$ dimensions, $\widetilde{Z}_{B}=1+g_{\mathrm{E}}^{2} N_{\mathrm{c}} \int_{p} \frac{T}{6\left(p^{2}+m_{\mathrm{E}}^{2}\right)^{2}}+g_{\mathrm{E}}^{4} N_{\mathrm{c}}^{2}\left[\frac{d^{3}-10 d^{2}+23 d-44}{3 d(d-5)(d-7)}-\frac{2 \lambda}{3}\right] \int_{p} \frac{T}{p^{2}+m_{\mathrm{E}}^{2}} \int_{q} \frac{T}{\left(q^{2}+m_{\mathrm{E}}^{2}\right)^{3}}+$ $\mathcal{O}\left(g_{\mathrm{E}}^{6} N_{\mathrm{c}}^{3}\right)$, where the integrals are given in eq. (D.1).
} 
perfectly. Our final "bare" expression reads 8

$$
\begin{aligned}
\delta \widetilde{\Gamma}_{\mathrm{MQCD}}^{(2)}[B]= & \frac{1}{2} B_{i}^{a}(q) B_{j}^{b}(r) \delta^{a b} \delta(q+r)\left(q^{2} \delta_{i j}-q_{i} q_{j}\right)\left(\frac{g_{\mathrm{E}}^{2} N_{\mathrm{c}} T \mu^{-2 \epsilon}}{16 \pi m_{\mathrm{E}}}\right)^{3}\left(\frac{\bar{\mu}}{2 m_{\mathrm{E}}}\right)^{6 \epsilon} \\
& \times\left\{\frac{1+4\left(\kappa_{2}-4 \lambda\right)}{6 \epsilon}+\frac{2\left(23510-12600 \zeta_{2}-1101 \ln 2\right)}{945}\right. \\
& \left.+\frac{4 \lambda+24 \lambda^{2}-\kappa_{1}(5-8 \ln 2)+\kappa_{2}(31-24 \ln 2)}{9}+\mathcal{O}(\epsilon)\right\}
\end{aligned}
$$

The $1 / \epsilon$-divergences in eq. (4.2) could a priori have an IR or UV origin. To find out, we have carried out the same computation by shielding all masses like in eq. (3.17), but with $m_{\mathrm{G}} \rightarrow m_{\mathrm{E}}$. Then only the divergence proportional to $4\left(\kappa_{2}-4 \lambda\right)$ remains. This indicates that the divergence not containing scalar self-couplings is purely of IR origin.

We can envisage two possible sources for the IR divergence. One is related to ultrasoft contributions of the same type as in section 3.3; these are analyzed in section 4.2. The other is related to the mass parameter $m_{\mathrm{E}}^{2}$. It is well known that the physical Debye mass, defined as a screening mass related to a "heavy-light" state, is non-perturbative starting at next-to-leading order $[38,39]$. Our $m_{\mathrm{E}}^{2}$ is not such a physical mass but rather a Lagrangian parameter. Nevertheless, $m_{\mathrm{E}}^{2}$ can still be considered IR sensitive at $\mathcal{O}\left(g_{\mathrm{ER}}^{4} T^{2}\right)$. Indeed, if we compute the 2-point function of $A_{0}^{a}$ at zero momentum, and shield all masses like in eq. (3.17), we find the UV divergence cancelled by the mass counterterm in eq. (2.9). In contrast, if we compute the 2-point function without IR-shielding, we find an additional $1 / \epsilon$-divergence proportional to $g_{\mathrm{ER}}^{4} T^{2}$, which depends on the gauge parameter $\alpha$. This is an IR divergence, i.e. $\sim g_{\mathrm{ER}}^{4} T^{2} / \epsilon_{\mathrm{IR}}$.

If we naively insert an ambiguity of this type into the 1-loop term in eq. (4.1) and re-expand up to 3 -loop order, the result is

$$
\frac{g_{\mathrm{ER}}^{2} N_{\mathrm{c}} T}{48 \pi\left[m_{\mathrm{ER}}^{2}+\frac{\beta}{\epsilon_{\mathrm{IR}}}\left(\frac{g_{\mathrm{ER}}^{2} N_{\mathrm{c}} T}{16 \pi}\right)^{2}\right]^{1 / 2}}-\frac{g_{\mathrm{ER}}^{2} N_{\mathrm{c}} T}{48 \pi m_{\mathrm{ER}}} \simeq-\frac{\beta}{6 \epsilon_{\mathrm{IR}}}\left(\frac{g_{\mathrm{ER}}^{2} N_{\mathrm{c}} T}{16 \pi m_{\mathrm{ER}}}\right)^{3} .
$$

On the non-perturbative level, $1 / \epsilon_{\mathrm{IR}}$ would turn into a multiple of $\ln \left(c m_{\mathrm{G}} / m_{\mathrm{ER}}\right)$, where $c$ is a non-perturbative constant and the scale $m_{\mathrm{G}}$ was defined around eq. (3.17).

Keeping in mind this expectation, we renormalize eq. (4.2) by employing the proper mass counterterm from eq. (2.9). The UV divergences proportional to $\kappa_{2}-4 \lambda$ duly cancel, and we find the 3-loop result

$$
\begin{aligned}
\widetilde{Z}_{B}^{(3)}+\delta \widetilde{Z}_{B}^{(3)}=\left(\frac{g_{\mathrm{ER}}^{2} N_{\mathrm{c}} T}{16 \pi m_{\mathrm{ER}}}\right)^{3}\{ & \frac{1}{6 \epsilon}+\left[1+\frac{8\left(\kappa_{2}-4 \lambda\right)}{3}\right] \ln \left(\frac{\bar{\mu}}{2 m_{\mathrm{ER}}}\right) \\
& +\frac{2\left(23510-12600 \zeta_{2}-1101 \ln 2\right)}{945} \\
& \left.+\frac{52 \lambda+24 \lambda^{2}-\kappa_{1}(5-8 \ln 2)+\kappa_{2}(19-24 \ln 2)}{9}\right\} .
\end{aligned}
$$

\footnotetext{
${ }^{8}$ The full $d$-dimensional form is given in appendix E, cf. eqs. (E.4)-(E.12).
} 


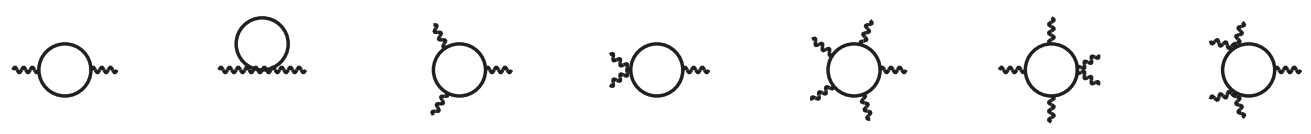

Figure 7. 1-loop contributions to the MQCD 2-point, 3-point and 5-point functions in the background field gauge. Wiggly lines denote ultrasoft gluons and solid lines adjoint scalars.

\subsection{Contribution from dimension-six operators in MQCD}

Parallelling section 3.3, let us finally consider contributions from ultrasoft effects to the gauge coupling, in the presence of dimension-six operators in MQCD. The action has the form in eq. (3.16), with the coefficients now completed to include the soft contribution:

$$
\mathcal{C}_{i}=\sum_{P}^{\prime} \frac{c_{i}}{P^{6}}+T \int_{p} \frac{\tilde{c}_{i}}{\left(p^{2}+m_{\mathrm{E}}^{2}\right)^{3}}, \quad i=1,3
$$

The spatial integral appearing is related to that in eq. (3.5) as shown by eq. (D.1),

$$
\int_{p} \frac{T}{\left(p^{2}+m_{\mathrm{E}}^{2}\right)^{3}}=\frac{m_{\mathrm{E}}^{d-6} \Gamma\left(3-\frac{d}{2}\right) T}{2(4 \pi)^{\frac{d}{2}}} \stackrel{3-2 \epsilon}{=} \frac{T \mu^{-2 \epsilon}}{32 \pi m_{\mathrm{E}}^{3}}\left[1+2 \epsilon\left(1+\ln \frac{\bar{\mu}}{2 m_{\mathrm{E}}}\right)+\mathcal{O}\left(\epsilon^{2}\right)\right] .
$$

Including the overall prefactor from eq. (3.16) and the integral from eq. (4.6), the new contributions to the coefficients of the dimension-six operators are $\sim g_{\mathrm{M}}^{2} T / m_{\mathrm{E}}^{3}$ at 1-loop level. Including a fictitious IR-regulator like in eq. (3.17), the 1-loop contribution from these operators to $\widetilde{Z}_{B}$ comes with a factor $\sim g_{\mathrm{M}}^{2} T m_{\mathrm{G}}$ and vanishes for $m_{\mathrm{G}} \rightarrow 0$, whereas the 2-loop contribution comes with a factor $\sim g_{\mathrm{M}}^{4} T^{2}$ and can yield a contribution $\sim g_{\mathrm{M}}^{6} T^{3} / m_{\mathrm{E}}^{3} \sim \mathcal{O}\left(g^{3}\right)$ to $\widetilde{Z}_{B}$. 2-loop contributions to the coefficients of dimension-six operators would be $\sim$ $g_{\mathrm{M}}^{4} T^{2} / m_{\mathrm{E}}^{4}$ and therefore lead to effects suppressed by $\sim \mathcal{O}\left(g^{4}\right)$. Dimension-eight operators, whose coefficients are $\sim g_{\mathrm{M}}^{2} T / m_{\mathrm{E}}^{5}$, lead to effects suppressed by $\sim g_{\mathrm{M}}^{10} T^{5} / m_{\mathrm{E}}^{5} \sim \mathcal{O}\left(g^{5}\right)$.

According to eq. (2.24), the value of $\tilde{c}_{1}$ can be inferred from the 2-point and that of $\tilde{c}_{3}$ from the 3-point vertex of the background field effective action. To be sure that no operators got overlooked, we have also determined them from the 5-point vertex, cf. the spatial part of eq. (2.27), which leads to several independent crosschecks (the diagrams are shown in figure 7 ). We find that the results are related in a curious way to the $d$-dependence of $c_{1}$ and $c_{3}$ in eq. (2.18): $:^{9,10}$

$$
\tilde{c}_{1}=-\frac{1}{120}, \quad \tilde{c}_{3}=-\frac{1}{180}
$$

\footnotetext{
${ }^{9}$ To our knowledge these values were first obtained for $d=3$ by P. Giovannangeli (unpublished, 2005), along lines that have recently been documented in ref. [40].

${ }^{10}$ We note in passing that even though the $\tilde{c}_{i}$ contribution in eq. (4.5) is parametrically larger by $\mathcal{O}\left(1 / g^{3}\right)$ than the $c_{i}$ contribution, the large value of $c_{1}$ in eq. (2.18) implies that numerically $c_{1}$ and $\tilde{c}_{1}$ give similar contributions if $g^{2} \sim 2$. If $g^{2} \gg 1, \mathcal{C}_{1}$ becomes positive.
} 
Inserting these values into eq. (3.20), and substituting $g_{\mathrm{M}}^{2}=g_{\mathrm{ER}}^{2} \mu^{2 \epsilon}(1+\mathcal{O}(g))$, we find a gauge-independent UV divergence and logarithmic part:

$$
\begin{aligned}
\delta \widetilde{\Gamma}_{\mathrm{IR}}^{(2)}[B]= & \frac{1}{2} B_{i}^{a}(q) B_{j}^{b}(r) \delta^{a b} \delta(q+r)\left(q^{2} \delta_{i j}-q_{i} q_{j}\right) \\
& \times\left(\frac{g_{\mathrm{ER}}^{2} N_{\mathrm{c}} T}{16 \pi m_{\mathrm{ER}}}\right)^{3}\left(-\frac{1}{45}\right)\left\{\frac{1}{\epsilon}+2 \ln \left(\frac{\bar{\mu}}{2 m_{\mathrm{ER}}}\right)+4 \ln \left(\frac{\bar{\mu}}{3 m_{\mathrm{G}}}\right)+\mathcal{O}(1)\right\} .
\end{aligned}
$$

This implies that the counterterm needed in MQCD reads $\delta \widetilde{Z}_{B}^{(3)}=\left(\frac{g_{\mathrm{ER}}^{2} N_{\mathrm{c}} T}{16 \pi m_{\mathrm{ER}}}\right)^{3} \frac{1}{45 \epsilon}$.

Obviously, eq. (4.8) does not match the divergence in eq. (4.4). In other words, if we subtract the part needed to serve as $\delta \widetilde{Z}_{B}^{(3)}$ from eq. (4.4), an IR divergence remains. In terms of the coefficient $\beta$ introduced in eq. (4.3), it amounts to $\beta=-\frac{13}{15}$. Let us stress that we have verified the gauge independence of this result. Therefore we are left to speculate that a nonperturbative mass ambiguity of the type discussed around eq. (4.3) prohibits a purely perturbative determination of $\widetilde{Z}_{B}^{(3)}$, and thus of $g_{\mathrm{M}}^{2}$ in terms of $g_{\mathrm{ER}}^{2}$ and $m_{\mathrm{ER}}$ at $\mathcal{O}\left(g_{\mathrm{ER}}^{6} T^{3} / m_{\mathrm{ER}}^{3}\right)$.

\section{Conclusions}

The main technical ingredient of this investigation was the analysis carried out in section 3 . We considered dimension-six operators induced by integrating out the "hard" momenta $\sim \pi T$ from thermal QCD [28]. Specifically, we computed at 1-loop and 2-loop levels the influence of these operators on the gauge coupling felt by ultrasoft (magnetostatic) modes. Remarkably, including UV divergences originating both from "soft" loops at the Debye scale $m_{\mathrm{E}} \sim g T$ and "ultrasoft" loops at the non-perturbative scale $\sim g^{2} T / \pi$, we observed an exact cancellation of the IR divergence found in a 3-loop determination of the EQCD gauge coupling (cf. eq. (2.12)) [22, 23]. This represents a nice crosscheck of the effective theory setup as a whole.

As a second technical ingredient, discussed in section 4, we considered the "soft" contributions to the ultrasoft gauge coupling. We determined direct 3-loop effects (cf. eq. (4.4)) and compared them with overlapping ultrasoft/soft contributions originating from dimension-six operators induced by integrating out the soft momenta $\sim m_{\mathrm{E}}$ (cf. eq. (4.8)). This time only a partial cancellation of soft IR divergences against ultrasoft/soft UV divergences was observed. As a culprit, we speculate that a non-perturbative ambiguity of the soft scale within EQCD sets an upper bound on the accuracy with which effects depending on $m_{\mathrm{E}}$ can be determined within perturbation theory. This may be surprising insofar as no such problem was met in 3-loop or 4-loop studies of the EQCD vacuum energy density $[15,31]$. However, the present quantity is different, being not directly a physical observable but rather an effective Lagrangian parameter (the MQCD gauge coupling $g_{\mathrm{M}}^{2}$ ).

On a more general level, the main conclusions that we draw are as follows:

(i) Even if the colour-electric scale $m_{\mathrm{E}} \sim g T$ is formally larger than the colour-magnetic scale $\sim g^{2} T / \pi$, it does play an essential role in the IR dynamics. Concretely, in terms of the IR divergence found by integrating out the hard scale $\sim \pi T$, the colour-electric scale is 1097 times more important than the colour-magnetic scale (cf. eq. (3.14)). 
(ii) Dimension-six operators need to be included in EQCD if good precision is required. Indeed, as we have demonstrated analytically (cf. point (i)), they do influence the IR dynamics of the system. This is a possible reason for why the super-renormalizable truncation of EQCD fails close to $T_{\mathrm{c}}$ even in pure Yang-Mills theory [19].

(iii) Apart from the indications in point (i) that the scale $m_{\mathrm{E}}$ is important, we also find trouble if we try to integrate it out. The reason could be that EQCD is a confining theory, and that physics at the scale $m_{\mathrm{E}}^{2}$ should in general be affected by non-perturbative ambiguities of $\mathcal{O}\left(g^{4} T^{2} / \pi^{2}\right)$. Once $m_{\mathrm{E}}$ is integrated out, some remnant of these ambiguities may remain, if the parameters of MQCD are determined up to the corresponding relative precision. It would be interesting to find a way to determine the leading non-perturbative contribution to $g_{\mathrm{M}}^{2}$ through lattice methods, even if this requires the simultaneous inclusion of the $1 / m_{\mathrm{E}}^{3}$-suppressed MQCD dimension-six operators in eq. (3.16).

\section{Acknowledgments}

This work was partly supported by the Swiss National Science Foundation (SNF) under grant 200020-168988, by the FONDECYT under project 1151281, and by the UBB under project GI-172309/C.

\section{A Spacetime and colour tensors}

Because the presence of a heat bath breaks Lorentz invariance, we need to introduce separate notation for spatial and zero spacetime indices. The full Kronecker symbol is denoted by

$$
\delta_{\mu \nu} \equiv T_{\mu \nu}+S_{\mu \nu}, \quad T_{\mu \nu} \equiv \delta_{\mu 0} \delta_{\nu 0}, \quad S_{\mu \nu} \equiv \delta_{\mu i} \delta_{\nu i} .
$$

We also introduce the totally symmetric tensors

$$
\begin{aligned}
T_{\mu \nu \rho \sigma} & \equiv \delta_{\mu 0} \delta_{\nu 0} \delta_{\rho 0} \delta_{\sigma 0} \\
T_{\mu \nu \rho \sigma \alpha \beta} & \equiv \delta_{\mu 0} \delta_{\nu 0} \delta_{\rho 0} \delta_{\sigma 0} \delta_{\alpha 0} \delta_{\beta 0} \\
\delta_{\mu \nu \rho \sigma} & \equiv \delta_{\mu \nu} \delta_{\rho \sigma}+2 \text { permutations } \\
\delta_{\mu \nu \rho \sigma \alpha \beta} & \equiv \delta_{\mu \nu} \delta_{\rho \sigma} \delta_{\alpha \beta}+14 \text { permutations } .
\end{aligned}
$$

For the colour indices, it is helpful to denote

$$
X^{a_{1} a_{2} \ldots a_{n}} \equiv f^{m_{n} a_{1} m_{1}} f^{m_{1} a_{2} m_{2}} \cdots f^{m_{n-1} a_{n} m_{n}},
$$

as well as the symmetrized versions

$$
X^{\left\{a_{1} \ldots a_{2}\right\} \ldots} \equiv \frac{1}{2}\left(X^{a_{1} \ldots a_{2} \ldots}+X^{a_{2} \ldots a_{1} \ldots}\right), \quad X^{\left[a_{1} \ldots a_{2}\right] \ldots} \equiv \frac{1}{2}\left(X^{a_{1} \ldots a_{2} \ldots}-X^{a_{2} \ldots a_{1} \ldots}\right) .
$$

These objects satisfy $X^{a_{n} a_{n-1} \ldots a_{2} a_{1}}=(-1)^{n} X^{a_{1} a_{2} \ldots a_{n-1} a_{n}}, X^{a_{1} a_{2} \ldots a_{n-1} a_{n}}=X^{a_{2} \ldots a_{n-1} a_{n} a_{1}}$. It follows that

$$
X^{\left\{a_{1} a_{2}\right\}\left[a_{3} a_{4}\right]}=X^{\left\{a_{1} a_{2}\right\}\left\{a_{3} a_{4} a_{5}\right\}}=X^{\left[a_{1} a_{2}\right]\left[a_{3} a_{4} a_{5}\right]}=X^{\left\{a_{1} a_{2} a_{3}\right\}\left[a_{4} a_{5} a_{6}\right]}=0 .
$$


Therefore we can write

$$
\begin{aligned}
X^{a_{1} a_{2} a_{3} a_{4}} & =X^{\left\{a_{1} a_{2}\right\}\left\{a_{3} a_{4}\right\}}+X^{\left[a_{1} a_{2}\right]\left[a_{3} a_{4}\right]}, \\
X^{a_{1} a_{2} a_{3} a_{4} a_{5}} & =X^{\left\{a_{1} a_{2}\right\}\left[a_{3} a_{4} a_{5}\right]}+X^{\left[a_{1} a_{2}\right]\left\{a_{3} a_{4} a_{5}\right\}}, \\
X^{a_{1} a_{2} a_{3} a_{4} a_{5} a_{6}} & =X^{\left\{a_{1} a_{2} a_{3}\right\}\left\{a_{4} a_{5} a_{6}\right\}}+X^{\left[a_{1} a_{2} a_{3}\right]\left[a_{4} a_{5} a_{6}\right]} .
\end{aligned}
$$

It may furthermore be noted that

$$
\begin{aligned}
X^{a_{1} a_{2} a_{3}} & =-\frac{N_{\mathrm{c}}}{2} f^{a_{1} a_{2} a_{3}}, \quad X^{\left[a_{1} a_{2}\right]\left[a_{3} a_{4}\right]}=-\frac{N_{\mathrm{c}}}{4} f^{m a_{1} a_{2}} f^{m a_{3} a_{4}}, \\
X^{\left[a_{1} a_{2}\right] a_{3}\left[a_{4} a_{5}\right]} & =-\frac{N_{\mathrm{c}}}{8} f^{m a_{1} a_{2}} f^{m a_{3} n} f^{n a_{4} a_{5}}, \\
f^{a_{1} a_{2} n} X^{n a_{3} a_{4} \cdots} & =2 X^{\left[a_{1} a_{2}\right] a_{3} a_{4} \cdots}=X^{a_{1} a_{2} a_{3} a_{4} \cdots}-X^{a_{2} a_{1} a_{3} a_{4} \cdots} .
\end{aligned}
$$

\section{B Basic sum-integrals}

Employing the notation defined in eqs. (A.1)-(A.5), the following relations can be established:

$$
\begin{aligned}
& \sum_{P}^{\prime} \frac{P_{\mu} P_{\nu}}{P^{4}}=\sum_{P}^{\prime} \frac{(1-d) T_{\mu \nu}+\delta_{\mu \nu}}{2 P^{2}}, \\
& \mathcal{F}_{P}^{\prime} \frac{P_{\mu} P_{\nu}}{P^{6}}=\mathcal{F}_{P}^{\prime} \frac{(3-d) T_{\mu \nu}+\delta_{\mu \nu}}{4 P^{4}}, \\
& \xi_{P}^{\prime} \frac{P_{\mu} P_{\nu}}{P^{8}}=\mathcal{F}_{P}^{\prime} \frac{(5-d) T_{\mu \nu}+\delta_{\mu \nu}}{6 P^{6}}, \\
& \mathcal{F}_{P}^{\prime} \frac{P_{\mu} P_{\nu} P_{\rho} P_{\sigma}}{P^{8}}=\mathcal{F}_{P}^{\prime}\left\{\frac{(3-d)(1-d) T_{\mu \nu \rho \sigma}}{24 P^{4}}\right. \\
& \left.+\frac{(3-d)\left(T_{\mu \nu} \delta_{\rho \sigma}+5 \text { permutations }\right)+\delta_{\mu \nu \rho \sigma}}{24 P^{4}}\right\}, \\
& \mathcal{F}_{P}^{\prime} \frac{P_{\mu} P_{\nu} P_{\rho} P_{\sigma}}{P^{10}}=\mathcal{F}_{P}^{\prime}\left\{\frac{(5-d)(3-d) T_{\mu \nu \rho \sigma}}{48 P^{6}}\right. \\
& \left.+\frac{(5-d)\left(T_{\mu \nu} \delta_{\rho \sigma}+5 \text { permutations }\right)+\delta_{\mu \nu \rho \sigma}}{48 P^{6}}\right\}, \\
& \mathcal{F}_{P}^{\prime} \frac{P_{\mu} P_{\nu} P_{\rho} P_{\sigma} P_{\alpha} P_{\beta}}{P^{12}}=\mathcal{F}_{P}^{\prime}\left\{\frac{(5-d)(3-d)(1-d) T_{\mu \nu \rho \sigma \alpha \beta}}{480 P^{6}}\right. \\
& +\frac{(5-d)(3-d)\left(T_{\mu \nu \rho \sigma} \delta_{\alpha \beta}+14 \text { permutations }\right)}{480 P^{6}} \\
& \left.+\frac{(5-d)\left(T_{\mu \nu} \delta_{\rho \sigma \alpha \beta}+14 \text { permutations }\right)+\delta_{\mu \nu \rho \sigma \alpha \beta}}{480 P^{6}}\right\} .
\end{aligned}
$$

These are needed for the computations in section 2.3. 


\section{Dimension-six vertices in the $S / T$ basis}

In section 2.3 we displayed (parts of) the vertices originating from eq. (2.13) in a basis in which spacetime indices appear in the form similar to appendix B. For the considerations of section 3, it is advantageous to employ a basis in which the spatial and temporal indices are strictly separated from each other. This can be implemented with the tensors $S_{\mu \nu \ldots}$ and $T_{\mu \nu \ldots}$, defined in eq. (A.1). In this section we display all the Chapman vertices originating from eq. (2.13) with such a notation.

The 2-point Chapman vertex reads

$$
\delta S_{\mathrm{EQCD}}^{(2)}=A_{\mu}^{a}(q) A_{\nu}^{a}(-q)\left(\oint_{P}^{\prime} \frac{g_{\mathrm{E}}^{2} N_{\mathrm{c}}}{P^{6}}\right)\left\{\eta_{1} q^{2}\left(q^{2} S_{\mu \nu}-q_{\mu} q_{\nu}\right)+\eta_{2} q^{4} T_{\mu \nu}\right\},
$$

where

$$
\eta_{1}=2 c_{1}, \quad \eta_{2}=2\left(c_{1}+c_{2}\right)
$$

The 3-point Chapman vertex becomes

$$
\begin{aligned}
\delta S_{\mathrm{EQCD}}^{(3)}= & A_{\mu}^{a}(q) A_{\nu}^{b}(r) A_{\rho}^{c}(s) f^{a b c} \delta(q+r+s)\left(\xi_{P}^{\prime} \frac{i g_{\mathrm{E}}^{3} N_{\mathrm{c}}}{P^{6}}\right) \\
& \times\left\{\xi_{1} q_{\mu} q_{\nu} q_{\rho}+\xi_{2} q_{\mu} q_{\nu} r_{\rho}+\xi_{3} q_{\mu} r_{\nu} q_{\rho}+\xi_{4} r_{\mu} q_{\nu} q_{\rho}\right. \\
& \left.+S_{\mu \nu}\left[\xi_{5} q^{2} q_{\rho}+\xi_{6} q^{2} r_{\rho}+\xi_{7} s^{2} q_{\rho}\right]+T_{\mu \nu}\left[\xi_{8} q^{2} q_{\rho}+\xi_{9} q^{2} r_{\rho}+\xi_{10} s^{2} q_{\rho}\right]\right\}
\end{aligned}
$$

where $q_{\mu} q_{\nu} q_{\rho}$ and $q_{\mu} q_{\nu} q_{\rho}+q_{\mu} q_{\nu} r_{\rho}-q_{\mu} r_{\nu} q_{\rho}=-q_{\mu}\left(q_{\nu} s_{\rho}+r_{\nu} q_{\rho}\right)$ actually vanish as can be seen by the relabelling $(r \leftrightarrow s, \nu \leftrightarrow \rho, b \leftrightarrow c)$. Therefore any change $\delta \xi_{1}$ or any simultaneous change $\delta \xi_{2}=-\delta \xi_{3}$ has no effect. It can be checked that eqs. (3.6) and (E.1)-(E.3) are invariant in these transformations. A representation of the coefficients can be chosen as

$$
\begin{aligned}
& \xi_{1}=0, \quad \xi_{2}=2 c_{3}, \quad \xi_{3}=-4 c_{1}, \quad \xi_{4}=-2 c_{3}, \\
& \xi_{5}=-3 c_{3}, \quad \xi_{6}=8 c_{1}-3 c_{3}, \quad \xi_{7}=3 c_{3}-4 c_{1}, \quad \xi_{8}=-4 c_{2}-3 c_{3}-c_{4}+c_{5}, \\
& \xi_{9}=8 c_{1}+4 c_{2}-3 c_{3}-c_{4}+c_{5}, \quad \xi_{10}=3 c_{3}-4 c_{1}+c_{4}-c_{5} .
\end{aligned}
$$

The 4-point vertex amounts to

$$
\begin{aligned}
\delta S_{\mathrm{EQCD}}^{(4)}=A_{\mu}^{a}(q) A_{\nu}^{b}(r) & A_{\alpha}^{c}(s) A_{\beta}^{d}(t) \delta(q+r+s+t)\left(\mathcal{f}_{P}^{\prime} \frac{g_{\mathrm{E}}^{4}}{P^{6}}\right) \\
\times\left\{X^{\{a b\}\{c d\}}\right. & {\left[S_{\mu \alpha} S_{\nu \beta}\left(\psi_{1} q^{2}+\psi_{3} q \cdot r\right)\right.} \\
& +T_{\mu \alpha} S_{\nu \beta}\left(\psi_{4} q^{2}+\psi_{5} r^{2}+\psi_{6} q \cdot r\right) \\
& +S_{\mu \nu} S_{\alpha \beta}\left(\psi_{10} q^{2}+\psi_{12} q \cdot r\right)+T_{\mu \nu} S_{\alpha \beta}\left(\psi_{13} q^{2}+\psi_{15} q \cdot r\right) \\
& +S_{\mu \nu} T_{\alpha \beta}\left(\psi_{16} q^{2}+\psi_{18} q \cdot r\right)+T_{\mu \nu \alpha \beta}\left(\psi_{19} q^{2}+\psi_{21} q \cdot r\right) \\
& +S_{\mu \alpha}\left(\psi_{22} q_{\nu} q_{\beta}+\psi_{23} q_{\nu} r_{\beta}+\psi_{24} r_{\nu} q_{\beta}+\psi_{25} r_{\nu} r_{\beta}\right) \\
& +T_{\mu \alpha}\left(\psi_{26} q_{\nu} q_{\beta}+\psi_{27} q_{\nu} r_{\beta}+\psi_{28} r_{\nu} q_{\beta}+\psi_{29} r_{\nu} r_{\beta}\right)
\end{aligned}
$$




$$
\begin{aligned}
& +S_{\mu \nu}\left(\psi_{30} q_{\alpha} q_{\beta}+\psi_{31} q_{\alpha} r_{\beta}\right)+T_{\mu \nu}\left(\psi_{34} q_{\alpha} q_{\beta}+\psi_{35} q_{\alpha} r_{\beta}\right) \\
& +S_{\alpha \beta}\left(\psi_{38} q_{\mu} q_{\nu}+\psi_{39} q_{\mu} r_{\nu}+\psi_{40} r_{\mu} q_{\nu}\right) \\
& \left.+T_{\alpha \beta}\left(\psi_{42} q_{\mu} q_{\nu}+\psi_{43} q_{\mu} r_{\nu}+\psi_{44} r_{\mu} q_{\nu}\right)\right] \\
& \left.+X^{[a b][c d]}\left[\psi_{i} \rightarrow \omega_{i}\right]\right\}
\end{aligned}
$$

where some coefficients have been dropped because they can be converted to the remaining ones through trivial renamings of indices and integration variables. The values are

$$
\begin{aligned}
\psi_{1} & =0, \quad \psi_{3}=-8 c_{1}, \\
\psi_{4} & =0, \quad \psi_{5}=0, \quad \psi_{6}=-16 c_{1}-4 c_{5}+8 c_{7}, \\
\psi_{10} & =-4 c_{1}, \quad \psi_{12}=-4 c_{1}, \\
\psi_{13} & =0, \quad \psi_{15}=0, \quad \psi_{16}=-8 c_{1}-2 c_{5}+4 c_{7}, \quad \psi_{18}=-8 c_{1}-2 c_{5}-4 c_{6}, \\
\psi_{19} & =-4 c_{1}-2 c_{5}+4 c_{7}+2 c_{9}, \quad \psi_{21}=-12 c_{1}-6 c_{5}-4 c_{6}+8 c_{7}-2 c_{8}+4 c_{9}, \\
\psi_{22} & =-8 c_{1}, \quad \psi_{23}=12 c_{1}, \quad \psi_{24}=-4 c_{1}, \quad \psi_{25}=4 c_{1}, \\
\psi_{26} & =-8 c_{1}-8 c_{2}, \quad \psi_{27}=12 c_{1}-20 c_{2}+8 c_{5}-16 c_{7}, \\
\psi_{28} & =-4 c_{1}+12 c_{2}-4 c_{5}+8 c_{7}, \quad \psi_{29}=4 c_{1}+4 c_{2}, \\
\psi_{30} & =4 c_{1}, \quad \psi_{31}=-4 c_{1}, \quad \psi_{34}=4 c_{1}+4 c_{2}, \quad \psi_{35}=-4 c_{1}-4 c_{2}, \\
\psi_{38} & =4 c_{1}, \quad \psi_{39}=0, \quad \psi_{40}=8 c_{1}, \\
\psi_{42} & =4 c_{1}-4 c_{2}+2 c_{5}-4 c_{7}, \quad \psi_{43}=8 c_{2}-2 c_{5}+4 c_{7}, \\
\psi_{44} & =8 c_{1}-8 c_{2}+4 c_{5}+4 c_{6}-4 c_{7}, \\
\omega_{1} & =-16 c_{1}, \quad \omega_{3}=8 c_{1}-12 c_{3}, \\
\omega_{4} & =-16 c_{1}-16 c_{2}, \quad \omega_{5}=-16 c_{1}-4 c_{5}+8 c_{7}, \\
\omega_{6} & =16 c_{1}-24 c_{3}-8 c_{4}+4 c_{5}+8 c_{7}, \\
\omega_{22} & =-24 c_{1}, \quad \omega_{23}=-44 c_{1}+24 c_{3}, \quad \omega_{24}=-12 c_{1}, \quad \omega_{25}=4 c_{1}, \\
\omega_{26} & =-24 c_{1}-24 c_{2}, \quad \omega_{27}=-44 c_{1}-12 c_{2}+24 c_{3}+8 c_{4}-8 c_{5} \\
\omega_{28} & =-12 c_{1}-28 c_{2}+4 c_{5}-8 c_{7}, \quad \omega_{29}=4 c_{1}-12 c_{2}+4 c_{5}-8 c_{7}, \\
\omega_{30} & =0 \\
\omega_{35} & =20 c_{1}+20 c_{2}-12 c_{3}-4 c_{4}+8 c_{7} .
\end{aligned}
$$

In the case of $\omega_{i}$, all coefficients associated with operators containing $S_{\alpha \beta}$ or $T_{\alpha \beta}$ vanish, because of antisymmetry.

The coefficients of the 4-point vertex listed above are not independent. Indeed momentum conservation leads to relations between the different structures defined in eq. (C.5), which implies that certain linear combinations of the coefficients couple to null operators. In the spirit of eq. (2.16), these ambiguities can be listed as transformations $\left(\Theta_{1} \ldots \Theta_{12}\right)$ whereby a simultaneous modification of the coefficients as indicated below has no physical 
meaning:

$$
\begin{aligned}
\Theta_{1}: & \delta \omega_{1}=-\delta \psi_{1}=\delta \psi_{10}, \\
\Theta_{2}: & \delta \omega_{4}=-\delta \psi_{4}=\delta \psi_{13}, \\
\Theta_{3}: & \delta \omega_{5}=-\delta \psi_{5}=\delta \psi_{16}, \\
\Theta_{4}: & \delta \omega_{22}=-\delta \psi_{22}=\delta \psi_{30}, \\
\Theta_{5}: & \delta \omega_{23}=-\delta \omega_{24}=\delta \omega_{31}=-\delta \psi_{23}=\delta \psi_{24}=2 \delta \psi_{39}=-2 \delta \psi_{40}, \\
\Theta_{6}: & \delta \omega_{25}=-\delta \psi_{25}=\delta \psi_{38}, \\
\Theta_{7}: & \delta \omega_{26}=-\delta \psi_{26}=\delta \psi_{34}, \\
\Theta_{8}: & \delta \omega_{27}=-\delta \omega_{28}=\delta \omega_{35}=-\delta \psi_{27}=\delta \psi_{28}=2 \delta \psi_{43}=-2 \delta \psi_{44} \\
\Theta_{9}: & \delta \omega_{29}=-\delta \psi_{29}=\delta \psi_{42}, \\
\Theta_{10}: & \delta \psi_{13}=\delta \psi_{15}=-\delta \psi_{16}=-\delta \psi_{18}, \\
\Theta_{11}: & \delta \psi_{30}=\delta \psi_{31}=-\delta \psi_{38}=-2 \delta \psi_{39}=-2 \delta \psi_{40}, \\
\Theta_{12}: & \delta \psi_{34}=\delta \psi_{35}=-\delta \psi_{42}=-2 \delta \psi_{43}=-2 \delta \psi_{44} .
\end{aligned}
$$

This list may not be complete. It can be checked that the expressions in eqs. (3.6) and (E.1)-(E.3) are invariant in these transformations.

The 5-point Chapman vertex reads

$$
\begin{aligned}
& \delta S_{\mathrm{EQCD}}^{(5)}= A_{\mu}^{a}(q) A_{\nu}^{b}(r) A_{\rho}^{c}(s) A_{\alpha}^{d}(t) A_{\beta}^{e}(u) \delta(q+r+s+t+u)\left(\sum_{P}^{\prime} \frac{i g_{\mathrm{E}}^{5} s_{\mu}}{P^{6}}\right) \\
& \times\left\{X ^ { \{ a b \} [ c d e ] } \left[\kappa_{1} S_{\rho \alpha} S_{\nu \beta}+\kappa_{2} S_{\rho \beta} S_{\nu \alpha}+\kappa_{3} S_{\rho \nu} S_{\alpha \beta}\right.\right. \\
&+\kappa_{4} T_{\rho \alpha} S_{\nu \beta}+\kappa_{5} T_{\rho \beta} S_{\nu \alpha}+\kappa_{6} T_{\rho \nu} S_{\alpha \beta} \\
&\left.+\kappa_{7} S_{\rho \alpha} T_{\nu \beta}+\kappa_{8} S_{\rho \beta} T_{\nu \alpha}+\kappa_{9} S_{\rho \nu} T_{\alpha \beta}+\kappa_{10} T_{\rho \nu \alpha \beta}\right] \\
&\left.+X^{[a b]\{c d e\}}\left[\kappa_{i} \rightarrow \lambda_{i}\right]\right\},
\end{aligned}
$$

where

$$
\begin{aligned}
\kappa_{1} & =-8 c_{1}, \quad \kappa_{2}=32 c_{1}, \quad \kappa_{3}=-8 c_{1}, \\
\kappa_{4} & =-8 c_{1}-8 c_{2}, \quad \kappa_{5}=32 c_{1}+32 c_{2}, \quad \kappa_{6}=-8 c_{1}-8 c_{2}, \\
\kappa_{7} & =-8 c_{1}-8 c_{2}, \quad \kappa_{8}=32 c_{1}+8 c_{5}-16 c_{7}, \quad \kappa_{9}=-8 c_{1}-8 c_{2}, \\
\kappa_{10} & =16 c_{1}+8 c_{5}-16 c_{7}-8 c_{9}, \\
\lambda_{1} & =40 c_{1}-24 c_{3}, \quad \lambda_{2}=-32 c_{1}+24 c_{3}, \quad \lambda_{3}=24 c_{1}, \\
\lambda_{4} & =40 c_{1}+8 c_{2}-24 c_{3}-8 c_{4}+8 c_{5}, \quad \lambda_{5}=-32 c_{1}+24 c_{3}+8 c_{4}-8 c_{5}, \\
\lambda_{6} & =24 c_{1}+24 c_{2}, \quad \lambda_{7}=40 c_{1}+8 c_{2}-24 c_{3}-8 c_{4}+8 c_{5}, \\
\lambda_{8} & =-32 c_{1}-32 c_{2}+24 c_{3}+8 c_{4}-16 c_{7}, \quad \lambda_{9}=24 c_{1}-8 c_{2}+8 c_{5}+16 c_{6}, \\
\lambda_{10} & =32 c_{1}+16 c_{5}+16 c_{6}-16 c_{7}+8 c_{8}-8 c_{9} .
\end{aligned}
$$


Finally the 6-point vertex can be expressed as

$$
\begin{aligned}
& \delta S_{\mathrm{EQCD}}^{(6)}=\int_{X} A_{\mu}^{a} A_{\nu}^{b} A_{\rho}^{c} A_{\sigma}^{d} A_{\alpha}^{e} A_{\beta}^{f} X^{a b c d e f}\left(\sum_{P}^{\prime} \frac{g_{\mathrm{E}}^{6}}{P^{6}}\right) \\
& \times\left\{\left[\chi_{1} S_{\rho \sigma} S_{\alpha \beta}+\chi_{2} S_{\rho \alpha} S_{\sigma \beta}+\chi_{3} S_{\rho \beta} S_{\sigma \alpha}\right] S_{\mu \nu}\right. \\
& +\left[\chi_{4} S_{\nu \alpha} S_{\rho \beta}+\chi_{5} S_{\nu \beta} S_{\rho \alpha}\right] S_{\mu \sigma} \\
& +\left[\chi_{6} S_{\rho \sigma} S_{\alpha \beta}+\chi_{7} S_{\rho \alpha} S_{\sigma \beta}+\chi_{8} S_{\rho \beta} S_{\sigma \alpha}\right] T_{\mu \nu} \\
& +\left[\chi_{9} S_{\nu \sigma} S_{\alpha \beta}+\chi_{10} S_{\nu \alpha} S_{\sigma \beta}\right] T_{\mu \rho} \\
& +\left[\chi_{11} S_{\nu \rho} S_{\alpha \beta}+\chi_{12} S_{\nu \alpha} S_{\rho \beta}+\chi_{13} S_{\nu \beta} S_{\rho \alpha}\right] T_{\mu \sigma} \\
& \left.+\chi_{14} S_{\mu \nu} T_{\rho \sigma \alpha \beta}+\chi_{15} S_{\mu \rho} T_{\nu \sigma \alpha \beta}+\chi_{16} S_{\mu \sigma} T_{\nu \rho \alpha \beta}+\chi_{17} T_{\mu \nu \rho \sigma \alpha \beta}\right\} \text {, }
\end{aligned}
$$

where

$$
\begin{aligned}
\chi_{1} & =-4 c_{1}+2 c_{3}, \quad \chi_{2}=16 c_{1}-6 c_{3}, \quad \chi_{3}=-4 c_{1}, \quad \chi_{4}=-2 c_{3}, \quad \chi_{5}=-8 c_{1}+6 c_{3}, \\
\chi_{6} & =-12 c_{1}-4 c_{2}+6 c_{3}+2 c_{4}-2 c_{5}, \quad \chi_{7}=16 c_{1}-6 c_{3}-2 c_{4}+4 c_{5}+4 c_{6}, \\
\chi_{8} & =-8 c_{1}-2 c_{5}-4 c_{6}, \\
\chi_{9} & =32 c_{1}+16 c_{2}-12 c_{3}-4 c_{4}+4 c_{5}, \quad \chi_{10}=-16 c_{1}+12 c_{3}+4 c_{4}-4 c_{5}, \\
\chi_{11} & =-4 c_{1}-4 c_{2}, \quad \chi_{12}=-6 c_{3}-2 c_{4}+4 c_{7}, \quad \chi_{13}=-8 c_{1}-8 c_{2}+6 c_{3}+2 c_{4}-4 c_{7}, \\
\chi_{14} & =-4 c_{1}-2 c_{5}-4 c_{6}-2 c_{8}, \quad \chi_{15}=16 c_{1}+8 c_{5}+8 c_{6}-8 c_{7}+4 c_{8}-4 c_{9}, \\
\chi_{16} & =-12 c_{1}-6 c_{5}-4 c_{6}+8 c_{7}-2 c_{8}+4 c_{9}, \quad \chi_{17}=-2 c_{10} .
\end{aligned}
$$

\section{Basic vacuum integrals}

For the computations of section 3 various $d$-dimensional vacuum integrals are needed. At 2-loop level their results can be expressed in terms of $H$ defined in eq. (3.9), multiplied by rational functions of $d$. For notational simplicity we denote the mass by $m$, let $\Delta_{p} \equiv p^{2}+m^{2}$, and omit the trivial factor $T$ included in eq. (3.9).

Making use of the integral

$$
\int_{p} \frac{1}{\Delta_{p}^{n}}=\frac{m^{d-2 n} \Gamma\left(n-\frac{d}{2}\right)}{(4 \pi)^{\frac{d}{2}} \Gamma(n)}
$$

factorized integrals can be expressed as

$$
\int_{p, q} \frac{m^{-2}}{\Delta_{p} \Delta_{q}}=-\frac{2(d-3) H}{d-2}, \quad \int_{p, q} \frac{1}{\Delta_{p}^{2} \Delta_{q}}=(d-3) H .
$$

A sunset integral with a power of the massless propagator reads

$$
\int_{p, q} \frac{1}{\Delta_{p} \Delta_{q}(p+q)^{2 n}}=\frac{m^{2 d-2 n-4} \Gamma\left(\frac{d}{2}-n\right) \Gamma(n+2-d) \Gamma^{2}\left(n+1-\frac{d}{2}\right)}{(4 \pi)^{d} \Gamma\left(\frac{d}{2}\right) \Gamma(2 n+2-d)} .
$$

In particular,

$$
\int_{p, q} \frac{1}{\Delta_{p} \Delta_{q}(p+q)^{2}}=H, \quad \int_{p, q} \frac{m^{2}}{\Delta_{p} \Delta_{q}(p+q)^{4}}=-\frac{(d-3) H}{2(d-5)} .
$$


A sunset integral with a power of a massive propagator reads

$$
\int_{p, q} \frac{1}{\Delta_{p}^{n} \Delta_{q}(p+q)^{2}}=\frac{m^{2 d-2 n-4} \Gamma\left(1-\frac{d}{2}\right) \Gamma\left(n+1-\frac{d}{2}\right)}{(d-n-2)(4 \pi)^{d} \Gamma(n)} .
$$

In particular,

$$
\int_{p, q} \frac{m^{2}}{\Delta_{p}^{2} \Delta_{q}(p+q)^{2}}=-\frac{(d-3) H}{2}, \quad \int_{p, q} \frac{m^{4}}{\Delta_{p}^{3} \Delta_{q}(p+q)^{2}}=\frac{(d-3)(d-4)(d-6) H}{8(d-5)} .
$$

Tensor integrals can be reduced to scalar integrals through

$$
\begin{aligned}
\left\langle p_{\mu} p_{\nu} p_{\alpha} p_{\beta}\right\rangle & =\frac{\left(S_{\mu \nu} S_{\alpha \beta}+S_{\mu \alpha} S_{\nu \beta}+S_{\mu \beta} S_{\nu \alpha}\right)\left\langle p^{4}\right\rangle}{d(d+2)} \\
\left\langle p_{\mu} p_{\nu} p_{\alpha} q_{\beta}\right\rangle & =\frac{\left(S_{\mu \nu} S_{\alpha \beta}+S_{\mu \alpha} S_{\nu \beta}+S_{\mu \beta} S_{\nu \alpha}\right)\left\langle p^{2} p \cdot q\right\rangle}{d(d+2)} \\
\left\langle p_{\mu} p_{\nu} q_{\alpha} q_{\beta}\right\rangle & =\frac{\left(S_{\mu \alpha} S_{\nu \beta}+S_{\mu \beta} S_{\nu \alpha}\right)\left\langle d(p \cdot q)^{2}-p^{2} q^{2}\right\rangle}{d(d-1)(d+2)}+\frac{S_{\mu \nu} S_{\alpha \beta}\left\langle(d+1) p^{2} q^{2}-2(p \cdot q)^{2}\right\rangle}{d(d-1)(d+2)},
\end{aligned}
$$

where $\langle\ldots\rangle$ represents a generic rotationally invariant expectation value, and $S_{\mu \nu} \equiv \delta_{\mu i} \delta_{\nu i}$.

In the considerations of section 3.3, another variant of the sunset integral was encountered,

$$
H_{3} \equiv \int_{p, q} \frac{1}{\Delta_{p} \Delta_{q} \Delta_{p+q}} .
$$

It can be written in terms of the hypergeometric function ${ }_{2} F_{1}[41,42]$,

$$
H_{3}=-\frac{3(d-2)}{4(d-3)}\left[{ }_{2} F_{1}\left(\frac{4-d}{2}, 1 ; \frac{5-d}{2} ; \frac{3}{4}\right)-3^{\frac{d-5}{2}} \frac{2 \pi \Gamma(5-d)}{\Gamma\left(\frac{4-d}{2}\right) \Gamma\left(\frac{6-d}{2}\right)}\right] \int_{p, q} \frac{m^{-2}}{\Delta_{p} \Delta_{q}} .
$$

At 3-loop level we need the values of two "basketball" integrals (cf. e.g. refs. [31, 37]):

$$
\begin{aligned}
B_{2} & \equiv \int_{p, q, r} \frac{1}{\Delta_{p} \Delta_{q}(p+r)^{2}(q+r)^{2}} \\
& =-\frac{m \mu^{-6 \epsilon}}{(4 \pi)^{3}}\left(\frac{\bar{\mu}}{2 m}\right)^{6 \epsilon}\left\{\frac{1}{2 \epsilon}+4+\epsilon\left[26+\frac{25 \zeta_{2}}{4}\right]+\mathcal{O}\left(\epsilon^{2}\right)\right\} \\
B_{4} & \equiv \int_{p, q, r} \frac{1}{\Delta_{p} \Delta_{q} \Delta_{p+r} \Delta_{q+r}} \\
& =-\frac{m \mu^{-6 \epsilon}}{(4 \pi)^{3}}\left(\frac{\bar{\mu}}{2 m}\right)^{6 \epsilon}\left\{\frac{1}{\epsilon}+8-4 \ln 2+\epsilon\left[52+\frac{17 \zeta_{2}}{2}-32 \ln 2+4 \ln ^{2} 2\right]+\mathcal{O}\left(\epsilon^{2}\right)\right\}
\end{aligned}
$$

\section{E Details concerning 2-loop and 3-loop results}

For completeness we report here technical results related to sections 3 and 4 that were too lengthy to fit the presentation in the main text. 
Consider first the coefficients $C_{1}, C_{2}$ and $C_{3}$, defined in eq. (3.10). Because of the general way in which we have parametrized the Chapman vertices (cf. appendix C), the expressions for these contain substantial "redundancies", which we reproduce here in full. This permits for very strong crosschecks, as discussed e.g. in the context of eqs. (C.7)(C.18) for the quartic Chapman vertex. The expressions read

$$
\begin{aligned}
C_{1}= & -\frac{8(d-1)\left[(2 d+3) \eta_{1}+2 d(d+2) \eta_{2}+(d+1) \xi_{5}-(d+2) \xi_{6}-\xi_{7}+d \xi_{10}\right]}{d-2} \\
& -\frac{8(d-1)\left[(d+1)(d+2) \xi_{8}-\left(d^{2}+3 d+1\right) \xi_{9}\right]}{d-2} \\
& +\frac{2(d-1)\left[4\left(\psi_{3}-\psi_{30}+\psi_{31}\right)-2(2 d+3) \psi_{10}+4 d \psi_{12}-3 \psi_{22}+\omega_{22}\right]}{d-2} \\
& -\frac{(d-1)\left[2\left(3 d^{2}-1\right) \psi_{4}+4\left(2 d^{2}+1\right)\left(\psi_{13}-\psi_{15}\right)+(5 d-1) \psi_{26}+d\left(\psi_{27}-\omega_{27}\right)\right]}{d-2} \\
& -\frac{(d-1)\left[\psi_{6}-\omega_{6}+\psi_{28}-\omega_{28}+2(5 d+1)\left(\psi_{34}-\psi_{35}\right)-2\left(d^{2}+3\right) \omega_{4}-(5 d+3) \omega_{26}\right]}{d-2} \\
& -\frac{(d-1)\left[(3 d+7)\left(\kappa_{4}+2 \psi_{1}\right)+(d-1)\left(2 \kappa_{5}-\lambda_{4}-2 \omega_{1}+2 \omega_{35}\right)-5 \kappa_{6}-(4 d+1) \lambda_{6}\right]}{d-2} \\
& -\frac{10 d(d-3)\left[\kappa_{10}-\lambda_{10}-4 \chi_{14}-2 \chi_{15}-2 \chi_{16}+4 \psi_{19}-2 \psi_{21}\right]}{d-2},
\end{aligned}
$$$$
C_{2}=\frac{2\left[18(d-1) \xi_{4}+(d+1)\left(d^{2}-9 d+12\right)\left(\xi_{6}-\xi_{5}\right)+12\left(d^{2}-3\right) \xi_{7}\right]}{3(d-5)}
$$$$
-\frac{2\left(d^{6}-13 d^{5}+49 d^{4}-83 d^{3}+208 d^{2}-114 d-156\right) \eta_{2}}{3(d-5)(d-7)}
$$$$
-\frac{\left(4 d^{5}-55 d^{4}+226 d^{3}-335 d^{2}+484 d-336\right) \xi_{8}}{3(d-5)(d-7)}
$$$$
+\frac{\left(4 d^{5}-55 d^{4}+226 d^{3}-323 d^{2}+388 d-252\right) \xi_{9}}{3(d-5)(d-7)}
$$$$
-\frac{4\left(d^{4}-10 d^{3}+25 d^{2}-51 d+51\right) \eta_{1}}{3(d-5)}-\frac{2\left(2 d^{4}-31 d^{3}+120 d^{2}-111 d+36\right) \xi_{10}}{3(d-5)}
$$$$
+\frac{(d-1)\left[(3 d+7) \psi_{1}-4\left(\psi_{3}-\psi_{30}+\psi_{31}\right)+2(2 d+3) \psi_{10}-4 d \psi_{12}+3 \psi_{22}\right]}{d-5}
$$$$
+\frac{(d-1)\left[\psi_{28}-2(d-1) \omega_{1}-2 \omega_{22}-\omega_{28}\right]}{2(d-5)}+\frac{d(37 d-39) \psi_{5}}{6}-\frac{d(3 d-1) \omega_{5}}{2}
$$$$
+\frac{(d-2)(d-3)(d-7)\left(\psi_{4}+3 \omega_{4}-2 \psi_{13}\right)}{6(d-5)}-\frac{\left(d^{3}-8 d^{2}+51 d-84\right) \psi_{6}}{12(d-5)}
$$$$
+\frac{2\left(d^{2}-8 d+9\right) \psi_{15}}{3(d-5)}+\frac{d(23 d-21) \psi_{16}}{3}-2\left(4 d^{2}-5 d+2\right) \psi_{18}
$$$$
+\frac{\left(d^{2}+7 d-12\right)\left(\psi_{26}-2 \psi_{34}+3 \omega_{26}\right)}{12}+\frac{d(d+1) \psi_{35}}{6}-2(d-2) \psi_{44}
$$$$
-\frac{\left(d^{3}-16 d^{2}+59 d-52\right) \omega_{6}}{4(d-5)}-\frac{(d-2)\left[\left(d^{2}-33\right) \psi_{27}-\left(d^{2}-24 d+87\right) \omega_{27}\right]}{12(d-5)}
$$ 


$$
\begin{aligned}
& +\frac{d(d-3)\left[5\left(\lambda_{10}-\kappa_{10}\right)-20(d-2) \psi_{19}+4(2 d-3) \psi_{21}-\omega_{35}\right]}{6} \\
& +\frac{\alpha(d-1)\left[\psi_{28}-\omega_{28}-2 \omega_{35}-8\left(2 \eta_{1}+\xi_{5}+\xi_{7}\right)\right]}{2(d-5)} \\
& +\frac{4 \alpha(d-1) \xi_{8}}{d-7}+\frac{8 \alpha(d-1)\left[(d-3) \eta_{2}-\xi_{9}\right]}{(d-5)(d-7)}, \\
C_{3}= & 8 d(d-1)\left[\eta_{2}+\xi_{8}+\xi_{10}\right] \\
& +\frac{4(d-1)\left[(d-1)\left(\eta_{1}+\xi_{5}\right)+2\left(\xi_{2}+\xi_{3}+\xi_{4}+\xi_{6}\right)+(d+1) \xi_{7}\right]}{d-5} \\
& +\frac{(d-1)\left[(3 d+7)\left(\psi_{1}+\psi_{25}\right)-4\left(\psi_{3}+\psi_{23}+\psi_{24}+\psi_{31}\right)+2(2 d+3)\left(\psi_{10}+\psi_{38}\right)\right]}{d-5} \\
& -\frac{(d-1)\left[4 d\left(\psi_{12}+\psi_{39}+\psi_{40}\right)-10\left(\psi_{22}+\psi_{30}\right)+(d-1)\left(\omega_{1}+\omega_{25}\right)\right]}{d-5} \\
& +2 d(d-1)\left[3\left(\psi_{5}+\psi_{29}\right)+4\left(\psi_{16}-\psi_{18}+\psi_{42}-\psi_{43}-\psi_{44}\right)-\omega_{5}-\omega_{29}\right] .
\end{aligned}
$$

After substituting the coefficients from appendix C, we get eq. (3.11).

As a second ingredient, we report the full $d$-dimensional version of eq. (4.2). The result can be expressed as

$$
\begin{aligned}
\delta \widetilde{\Gamma}_{\mathrm{MQCD}}^{(2)}[B]= & \frac{1}{2} B_{i}^{a}(q) B_{j}^{b}(r) \delta^{a b} \delta(q+r)\left(q^{2} \delta_{i j}-q_{i} q_{j}\right)\left(\frac{g_{\mathrm{E}}^{2} N_{\mathrm{c}}}{m_{\mathrm{E}}^{2}}\right)^{3} \\
& \times\left\{\left(r_{1}+\tilde{r}_{1}\right)(d) I^{3}\left(m_{\mathrm{E}}\right)+r_{2}(d) m_{\mathrm{E}}^{2} B_{2}\left(m_{\mathrm{E}}\right)+\left(r_{3}+\tilde{r}_{3}\right)(d) m_{\mathrm{E}}^{2} B_{4}\left(m_{\mathrm{E}}\right)\right\}
\end{aligned}
$$

where the pure gauge contributions are parametrized by

$$
\begin{aligned}
& r_{1}(d)=-\frac{(d-2) p_{1}(d)}{384(d-10)(d-8)(d-7)(d-6)(d-5)(d-4)(d-3)^{2}(d-1) d}, \\
& r_{2}(d)=\frac{(3 d-10)(3 d-8) p_{2}(d)}{128(d-3)(d-1) d(2 d-11)(2 d-9)(2 d-7)}, \\
& r_{3}(d)=\frac{(3 d-10)(3 d-8) p_{3}(d)}{256(d-10)(d-8)(d-6)(d-4)(d-1) d},
\end{aligned}
$$

with the non-factorizable polynomials

$$
\begin{aligned}
p_{1}(d)= & 12 d^{12}-628 d^{11}+14447 d^{10}-193505 d^{9}+1689420 d^{8}-10234582 d^{7} \\
& +44883931 d^{6}-147059385 d^{5}+366585830 d^{4}-689809244 d^{3} \\
& +929595256 d^{2}-791686464 d+314842752 \\
p_{2}(d)= & 12 d^{7}-308 d^{6}+3175 d^{5}-17441 d^{4}+57347 d^{3} \\
& -117419 d^{2}+138786 d-70872 \\
p_{3}(d)= & 3 d^{5}-60 d^{4}+359 d^{3}-670 d^{2}+400 d+736
\end{aligned}
$$

where $I, B_{2}$ and $B_{4}$ are the master integrals from eqs. (3.5), (D.12) and (D.13), respectively. 
In terms of the couplings from eqs. (2.5)-(2.7), the scalar contributions amount to

$$
\begin{aligned}
\tilde{r}_{1}(d)=\frac{d-2}{8}\{ & \left\{\frac{(d-4)\left(3 d^{5}-49 d^{4}+283 d^{3}-779 d^{2}+1238 d-1056\right) \lambda}{3(d-7)(d-5)(d-3) d}\right. \\
& -\frac{(d-4)(3 d-10) \lambda^{2}}{3} \\
& \left.+\frac{(d-2)^{2}\left(9 d^{2}-77 d+158\right) \kappa_{1}}{16(d-6)(d-4)(d-3) d}+\frac{(d-10)(d-2)^{2} \kappa_{2}}{16(d-4) d}\right\}, \\
\tilde{r}_{3}(d)= & \frac{(3 d-10)(3 d-8)\left(d^{2}-5 d-2\right)\left[\kappa_{1}+(d-6) \kappa_{2}\right]}{256(d-6)(d-4) d} .
\end{aligned}
$$

Setting $d=3-2 \epsilon$, inserting the values of the master integrals, and carrying out a Taylor expansion in $\epsilon$, eq. (E.4) goes over into eq. (4.2).

Open Access. This article is distributed under the terms of the Creative Commons Attribution License (CC-BY 4.0), which permits any use, distribution and reproduction in any medium, provided the original author(s) and source are credited.

\section{References}

[1] P.H. Ginsparg, First order and second order phase transitions in gauge theories at finite temperature, Nucl. Phys. B 170 (1980) 388 [inSPIRE].

[2] T. Appelquist and R.D. Pisarski, High-temperature Yang-Mills theories and three-dimensional quantum chromodynamics, Phys. Rev. D 23 (1981) 2305 [INSPIRE].

[3] K. Kajantie, M. Laine, K. Rummukainen and M.E. Shaposhnikov, Generic rules for high temperature dimensional reduction and their application to the Standard Model, Nucl. Phys. B 458 (1996) 90 [hep-ph/9508379] [INSPIRE].

[4] T. Brauner, T.V.I. Tenkanen, A. Tranberg, A. Vuorinen and D.J. Weir, Dimensional reduction of the Standard Model coupled to a new singlet scalar field, JHEP 03 (2017) 007 [arXiv: 1609.06230] [INSPIRE].

[5] L. Niemi, H.H. Patel, M.J. Ramsey-Musolf, T.V.I. Tenkanen and D.J. Weir, Electroweak phase transition in the $\Sigma S M-I$ : dimensional reduction, arXiv:1802.10500 [INSPIRE].

[6] S. Caron-Huot, $O(g)$ plasma effects in jet quenching, Phys. Rev. D 79 (2009) 065039 [arXiv:0811.1603] [INSPIRE].

[7] M. Panero, K. Rummukainen and A. Schäfer, Lattice study of the jet quenching parameter, Phys. Rev. Lett. 112 (2014) 162001 [arXiv:1307.5850] [INSPIRE].

[8] M. D'Onofrio, A. Kurkela and G.D. Moore, Renormalization of null Wilson lines in EQCD, JHEP 03 (2014) 125 [arXiv:1401.7951] [INSPIRE].

[9] J. Ghiglieri, J. Hong, A. Kurkela, E. Lu, G.D. Moore and D. Teaney, Next-to-leading order thermal photon production in a weakly coupled quark-gluon plasma, JHEP 05 (2013) 010 [arXiv: 1302.5970] [INSPIRE].

[10] J. Ghiglieri and G.D. Moore, Low mass thermal dilepton production at NLO in a weakly coupled quark-gluon plasma, JHEP 12 (2014) 029 [arXiv: 1410.4203] [INSPIRE]. 
[11] J. Ghiglieri and M. Laine, Neutrino dynamics below the electroweak crossover, JCAP 07 (2016) 015 [arXiv: 1605.07720] [INSPIRE].

[12] A.D. Linde, Infrared problem in thermodynamics of the Yang-Mills gas, Phys. Lett. B 96 (1980) 289 [inSPIRE].

[13] D.J. Gross, R.D. Pisarski and L.G. Yaffe, $Q C D$ and instantons at finite temperature, Rev. Mod. Phys. 53 (1981) 43 [InSPIRE].

[14] S. Nadkarni, Dimensional reduction in hot QCD, Phys. Rev. D 27 (1983) 917 [InSPIRE].

[15] E. Braaten and A. Nieto, Free energy of QCD at high temperature, Phys. Rev. D 53 (1996) 3421 [hep-ph/9510408] [INSPIRE].

[16] M. Laine, A non-perturbative contribution to jet quenching, Eur. Phys. J. C 72 (2012) 2233 [arXiv: 1208.5707] [INSPIRE].

[17] B. Svetitsky and L.G. Yaffe, Critical behavior at finite temperature confinement transitions, Nucl. Phys. B 210 (1982) 423 [InSPIRE].

[18] K. Kajantie, M. Laine, A. Rajantie, K. Rummukainen and M. Tsypin, The phase diagram of three-dimensional SU(3) + adjoint Higgs theory, JHEP 11 (1998) 011 [hep-lat/9811004] [INSPIRE].

[19] A. Hietanen, K. Kajantie, M. Laine, K. Rummukainen and Y. Schröder, Three-dimensional physics and the pressure of hot QCD, Phys. Rev. D 79 (2009) 045018 [arXiv:0811.4664] [INSPIRE].

[20] M. Laine and Y. Schröder, Two-loop QCD gauge coupling at high temperatures, JHEP 03 (2005) 067 [hep-ph/0503061] [INSPIRE].

[21] I. Ghisoiu, J. Möller and Y. Schröder, Debye screening mass of hot Yang-Mills theory to three-loop order, JHEP 11 (2015) 121 [arXiv:1509.08727] [INSPIRE].

[22] I. Ghisoiu, Three-loop Debye mass and effective coupling in thermal QCD, Ph.D. thesis, University of Bielefeld, Bielefeld, Germany, (2013)

[https://pub.uni-bielefeld.de/publication/2632705].

[23] I. Ghisoiu and Y. Schröder, Three-loop Debye mass and effective coupling in thermal QCD, poster presentation at the Latsis EPFL Symposium Strong and Electroweak Matter (SEWM14), Lausanne, Switzerland, 14-18 July 2014 [http://www.sewm14.unibe.ch/ghisoiu.pdf].

[24] K. Farakos, K. Kajantie, K. Rummukainen and M.E. Shaposhnikov, 3D physics and the electroweak phase transition: perturbation theory, Nucl. Phys. B 425 (1994) 67 [hep-ph/9404201] [INSPIRE].

[25] M. Laine and A. Rajantie, Lattice continuum relations for $3 d \mathrm{SU}(N)+$ Higgs theories, Nucl. Phys. B 513 (1998) 471 [hep-lat/9705003] [INSPIRE].

[26] L.F. Abbott, The background field method beyond one loop, Nucl. Phys. B 185 (1981) 189 [INSPIRE].

[27] J. Möller and Y. Schröder, Three-loop matching coefficients for hot QCD: reduction and gauge independence, JHEP 08 (2012) 025 [arXiv:1207.1309] [INSPIRE].

[28] S. Chapman, A new dimensionally reduced effective action for $Q C D$ at high temperature, Phys. Rev. D 50 (1994) 5308 [hep-ph/9407313] [INSPIRE]. 
[29] E. Megías, E. Ruiz Arriola and L.L. Salcedo, The thermal heat kernel expansion and the one loop effective action of QCD at finite temperature, Phys. Rev. D 69 (2004) 116003 [hep-ph/0312133] [INSPIRE].

[30] J.C. Collins and J.A.M. Vermaseren, Axodraw version 2, arXiv:1606.01177 [INSPIRE].

[31] K. Kajantie, M. Laine, K. Rummukainen and Y. Schröder, Four loop vacuum energy density of the $\mathrm{SU}\left(N_{c}\right)+$ adjoint Higgs theory, JHEP 04 (2003) 036 [hep-ph/0304048] [INSPIRE].

[32] P. Giovannangeli, Two loop renormalization of the magnetic coupling in hot QCD, Phys. Lett. B 585 (2004) 144 [hep-ph/0312307] [INSPIRE].

[33] P. Nogueira, Automatic Feynman graph generation, J. Comput. Phys. 105 (1993) 279 [INSPIRE].

[34] S. Laporta, High precision calculation of multiloop Feynman integrals by difference equations, Int. J. Mod. Phys. A 15 (2000) 5087 [hep-ph/0102033] [inSPIRE].

[35] A. von Manteuffel and C. Studerus, Reduze 2 - distributed Feynman integral reduction, arXiv:1201.4330 [INSPIRE].

[36] J. Kuipers, T. Ueda, J.A.M. Vermaseren and J. Vollinga, FORM version 4.0, Comput. Phys. Commun. 184 (2013) 1453 [arXiv:1203.6543] [InSPIRE].

[37] A.K. Rajantie, Feynman diagrams to three loops in three-dimensional field theory, Nucl. Phys. B 480 (1996) 729 [Erratum ibid. B 513 (1998) 761] [hep-ph/9606216] [INSPIRE].

[38] A.K. Rebhan, The non-Abelian Debye mass at next-to-leading order, Phys. Rev. D 48 (1993) R3967 [hep-ph/9308232] [InSPIRE].

[39] P.B. Arnold and L.G. Yaffe, The non-Abelian Debye screening length beyond leading order, Phys. Rev. D 52 (1995) 7208 [hep-ph/9508280] [INSPIRE].

[40] C.P. Korthals Altes, The unbearable smallness of magnetostatic QCD corrections, arXiv: 1801.00019 [INSPIRE].

[41] A.I. Davydychev and J.B. Tausk, Two loop selfenergy diagrams with different masses and the momentum expansion, Nucl. Phys. B 397 (1993) 123 [INSPIRE].

[42] Y. Schröder and A. Vuorinen, High-precision $\epsilon$-expansions of single-mass-scale four-loop vacuum bubbles, JHEP 06 (2005) 051 [hep-ph/0503209] [INSPIRE]. 